\title{
Analytical theory and nonlinear $\delta f$ perturbative simulations of temperature anisotropy instability in intense charged particle beams
}

\author{
Edward A. Startsev, Ronald C. Davidson, and Hong Qin \\ Plasma Physics Laboratory, Princeton University, Princeton, New Jersey 08543, USA
}

(Received 8 May 2003; published 15 August 2003)

\begin{abstract}
In plasmas with strongly anisotropic distribution functions $\left(T_{\| b} / T_{\perp b} \ll 1\right)$ a Harris-like collective instability may develop if there is sufficient coupling between the transverse and longitudinal degrees of freedom. Such anisotropies develop naturally in accelerators and may lead to a deterioration of beam quality. This paper extends previous numerical studies [E. A. Startsev, R. C. Davidson, and H. Qin, Phys. Plasmas 9, 3138 (2002)] of the stability properties of intense non-neutral charged particle beams with large temperature anisotropy $\left(T_{\perp b} \gg T_{\| b}\right)$ to allow for nonaxisymmetric perturbations with $\partial / \partial \theta \neq 0$. The most unstable modes are identified, and their eigenfrequencies, radial mode structure, and nonlinear dynamics are determined. The simulation results clearly show that moderately intense beams with $s_{b}=\hat{\omega}_{p b}^{2} / 2 \gamma_{b}^{2} \omega_{\beta \perp}^{2} \gtrsim 0.5$ are linearly unstable to short-wavelength perturbations with $k_{z}^{2} r_{b}^{2} \gtrsim 1$, provided the ratio of longitudinal and transverse temperatures is smaller than some threshold value. Here, $\hat{\omega}_{p b}^{2}=4 \pi \hat{n}_{b} e_{b}^{2} / \gamma_{b} m_{b}$ is the relativistic plasma frequency squared, and $\omega_{\beta \perp}$ is the betatron frequency associated with the applied smooth-focusing field. A theoretical model is developed based on the Vlasov-Maxwell equations which describes the essential features of the linear stages of instability. Both the simulations and the analytical theory predict that the dipole mode (azimuthal mode number $m=1$ ) is the most unstable mode. In the nonlinear stage, tails develop in the longitudinal momentum distribution function, and the kinetic instability saturates due to resonant wave-particle interactions.
\end{abstract}

DOI: 10.1103/PhysRevSTAB.6.084401

\section{INTRODUCTION}

Periodic focusing accelerators, transport systems, and storage rings [1-5] have a wide range of applications ranging from basic scientific research in high energy and nuclear physics, to applications such as heavy ion fusion, spallation neutron sources, tritium production, and nuclear waste transmutation, to mention a few examples. Of particular importance at the high beam currents and charge densities of practical interest are the effects of the intense self-fields produced by the beam space charge and current on determining the detailed equilibrium, stability, and transport properties. While considerable progress has been made in understanding the self-consistent evolution of the beam distribution function, $f_{b}(\mathbf{x}, \mathbf{p}, t)$, and self-generated electric and magnetic fields, $\mathbf{E}^{s}(\mathbf{x}, t)$ and $\mathbf{B}^{s}(\mathbf{x}, t)$, in kinetic analyses based on the nonlinear Vlasov-Maxwell equations [1,6-10], in numerical simulation studies of intense beam propagation [11-21], and in macroscopic warm-fluid models [22-25], the effects of finite geometry and space-charge effects often make predictions of detailed stability behavior difficult. It is therefore important to develop an improved understanding of fundamental collective stability properties, including the case where a large temperature anisotropy $\left(T_{\perp b} \gg T_{\| b}\right)$ can drive a Harris-like instability $[26,27]$, familiar in the study of electrically neutral plasmas.

It is well known that in neutral plasmas with strongly anisotropic distributions $\left(T_{\| b} / T_{\perp b} \ll 1\right)$ a collective instability may develop if there is sufficient coupling between the transverse and longitudinal degrees of freedom
PACS numbers: 41.75.-i, 52.27.Jt, 52.59.Sa, 29.27.-a

$[26,27]$. Such anisotropies develop naturally in accelerators. Indeed, due to the conservation of energy for particles with charge $e_{b}$ and mass $m_{b}$ accelerated by a voltage $V$, the energy spread of particles in the beam does not change, and (nonrelativistically) $\Delta E_{b i}=m_{b} \Delta v_{b i}^{2} / 2=$ $\Delta E_{b f}=m_{b} V_{b} \Delta v_{b f}$, where $V_{b}=\left(e_{b} V / m_{b}\right)^{1 / 2}$ is the average beam velocity after acceleration. Therefore, the velocity spread squared, or equivalently the temperature, changes according to $T_{\| b f}=T_{\| b i}^{2} / 2 e_{b} V$ (for a nonrelativistic beam). At the same time, the transverse temperature may increase due to nonlinearities in the applied and selffield forces, nonstationary beam profiles, and beam mismatch. These processes provide the free energy to drive collective instabilities and may lead to a deterioration of beam quality $[20,28,29]$. The instability may also lead to an increase of longitudinal velocity spread, which will make the focusing of the beam difficult and may impose a limit on the minimum spot size achievable in heavy ion fusion experiments.

Previous studies of this anisotropy-driven instability for long, coasting beams $[8,11,12,23,30-34]$ have shown that moderately intense beams with normalized beam intensity $s_{b}=\omega_{p b}^{2} / 2 \gamma_{b}^{2} \omega_{f}^{2} \gtrsim 0.5$ are linearly unstable to short-wavelength, axisymmetric $(\partial / \partial \theta=0)$ perturbations with $k_{z}^{2} r_{b}^{2} \geqslant 1$, provided the ratio of longitudinal to transverse temperatures is smaller than some threshold value. Here, $\omega_{p b}^{2}=4 \pi \hat{n}_{b} e_{b}^{2} / \gamma_{b} m_{b}$ is the relativistic plasma frequency squared, $\hat{n}_{b}$ is the on-axis number density of beam particles, $\gamma_{b}=\left(1-V_{b}^{2} / c^{2}\right)^{-1 / 2}$ is the relativistic mass factor, $V_{b}=\beta_{b} c$ is the average beam velocity in the axial direction, and $\omega_{f}=$ const is the 
smooth-focusing frequency associated with the applied field. Detailed stability properties for a finite-length charge bunch have been investigated by Hofmann et al. $[35,36]$. In this article, we extend our previous analytical and numerical studies [11] of this instability to the case of nonaxisymmetric perturbations with $\partial / \partial \theta \neq 0$. A simplified kinetic theory of the instability for arbitrary three-dimensional perturbations about a bi-Maxwellian distribution is presented in this paper, which appears to capture the main features of the instability and is a relatively straightforward generalization of the analysis of the Harris instability in electrically neutral plasma to the case of an intense particle beam with intense selffields. The 3D nonlinear perturbative particle simulation code $[15,17]$, called the Beam Equilibrium, Stability and Transport (BEST) code, is used to systematically study the electrostatic stability properties of intense non-neutral charged particle beams with large temperature anisotropy $\left(T_{\perp b} \gg T_{\| b}\right)$. The most unstable modes are identified, and their eigenfrequencies and radial mode structure are determined for perturbations with general azimuthal mode number $m$. The instability thresholds obtained in the simulations as well as detailed simulations of the nonlinear development and saturation of the instability are presented. We identify the main saturation mechanism as quasilinear stabilization due to resonant wave-particle interactions (Landau damping).

The organization of this paper is the following. In Sec. II, we present a simplified kinetic model of the instability based on a matrix dispersion equation derived from the linearized Vlasov-Poisson equations. The nonlinear $\delta f$ simulation method is briefly described in Sec. III, and in Sec. IV we present detailed simulation results for a wide range of system parameters and perturbations with general azimuthal mode number $m$.

\section{LINEAR STABILITY THEORY}

\section{Kinetic description}

It is important to extend theoretical studies of the kinetic stability properties of anisotropic beams to distribution functions other than the KapchinskijVladimirskij (KV) distribution [6]. This is because the $\mathrm{KV}$ distribution has an (unphysical) inverted population in transverse phase-space variables, which provides the free energy to drive collective instabilities at sufficiently high beam intensity that are intrinsic to this inverted population [7,8]. This, of course, can mask the effects of anisotropy-driven instabilities. To this end, we briefly outline here a simple derivation of the Harris-like insta- bility [26,27] in intense particle beams for electrostatic perturbations about the thermal equilibrium distribution with temperature anisotropy $\left(T_{\perp b}>T_{\| b}\right)$ described in the beam frame $\left(V_{b}=0\right.$ and $\left.\gamma_{b}=1\right)$ by the self-consistent axisymmetric Vlasov equilibrium $[1,10]$

$$
\begin{aligned}
f_{b}^{0}(r, \mathbf{p})= & \frac{\hat{n}_{b}}{\left(2 \pi m_{b} T_{\perp b}\right)} \exp \left(-\frac{H_{\perp}}{T_{\perp b}}\right) \frac{1}{\left(2 \pi m_{b} T_{\| b}\right)^{1 / 2}} \\
& \times \exp \left(-\frac{p_{z}^{2}}{2 m_{b} T_{\| b}}\right) .
\end{aligned}
$$

Here, $\quad H_{\perp}=p_{\perp}^{2} / 2 m_{b}+(1 / 2) m_{b} \omega_{f}^{2}\left(x^{2}+y^{2}\right)+e_{b} \phi^{0}(r)$ is the single-particle Hamiltonian for transverse particle motion, $p_{\perp}=\left(p_{x}^{2}+p_{y}^{2}\right)^{1 / 2}$ is the transverse particle momentum, $r=\left(x^{2}+y^{2}\right)^{1 / 2}$ is the radial distance from the beam axis, $\omega_{f}=$ const is the transverse frequency associated with the applied focusing field in the smoothfocusing approximation, and $\phi^{0}(r)$ is the equilibrium space-charge potential determined self-consistently from Poisson's equation,

$$
\frac{1}{r} \frac{\partial}{\partial r} r \frac{\partial \phi^{0}}{\partial r}=-4 \pi e_{b} n_{b}^{0}
$$

where $n_{b}^{0}(r)=\int d^{3} p f_{b}^{0}(r, \mathbf{p})$ is the equilibrium number density of beam particles. For simplicity, the analysis is carried out in the beam frame $\left(V_{b}=0\right.$ and $\left.\gamma_{b}=1\right)$. Furthermore, setting $\phi^{0}(r=0)=0$, the constant $\hat{n}_{b}$ occurring in Eq. (1) can be identified with the on-axis density $n_{b}^{0}(r=0)$, and the constants $T_{\perp b}$ and $T_{\| b}$ can be identified with the transverse and longitudinal temperatures (energy units), respectively.

For present purposes, we consider small-amplitude electrostatic perturbations of the form

$$
\delta \phi(\mathbf{x}, t)=\hat{\delta \phi}(r) \exp \left(i m \theta+i k_{z} z-i \omega t\right),
$$

where $\delta \phi(\mathbf{x}, t)$ is the perturbed electrostatic potential, $k_{z}$ is the axial wave number, $m$ is the azimuthal mode number, and $\omega$ is the complex oscillation frequency, with $\operatorname{Im} \omega>0$ corresponding to instability (temporal growth). Without presenting algebraic details, using the method of characteristics [1], the linearized Poisson equation can be expressed as

$$
\begin{aligned}
& \frac{1}{r} \frac{\partial}{\partial r} r \frac{\partial}{\partial r} \hat{\delta} \hat{\phi}(r)-k_{z}^{2} \hat{\boldsymbol{\phi}} \hat{\phi}(r)-\frac{m^{2}}{r^{2}} \hat{\delta} \hat{\phi}(r) \\
& \quad=-4 \pi e_{b} \int d^{3} p \delta \hat{f}_{b}(r, \mathbf{p})
\end{aligned}
$$

where

$$
\delta \hat{f}_{b}(r, \mathbf{p})=e_{b} \frac{\partial f_{b}^{0}}{\partial H_{\perp}} \hat{\boldsymbol{\phi} \phi}+e_{b}\left[\left(\omega-k_{z} v_{z}\right) \frac{\partial f_{b}^{0}}{\partial H_{\perp}}+k_{z} v_{z} \frac{\partial f_{b}^{0}}{\partial H_{\|}}\right] i \int_{-\infty}^{t} d t^{\prime} \hat{\boldsymbol{\phi}}\left[r^{\prime}\left(t^{\prime}\right)\right] \exp \left\{i\left(k_{z} \boldsymbol{v}_{z}-\omega\right)\left(t^{\prime}-t\right)+i m\left[\theta^{\prime}\left(t^{\prime}\right)-\theta^{\prime}(t)\right]\right\}
$$


for perturbations about the choice of the anisotropic thermal equilibrium distribution function in Eq. (1). Here, $H_{\|}=p_{z}^{2} / 2 m_{b}$ and $v_{z}=p_{z} / m_{b}$. In the orbit integral in Eq. (5), $\operatorname{Im} \omega>0$ is assumed, and $r^{\prime}\left(t^{\prime}\right)=$ $\left[x^{\prime 2}\left(t^{\prime}\right)+y^{\prime 2}\left(t^{\prime}\right)\right]^{1 / 2}$ and $\theta^{\prime}\left(t^{\prime}\right)$ are the transverse orbits in the equilibrium field configuration such that $\left[\mathbf{x}_{\perp}^{\prime}\left(t^{\prime}\right), \mathbf{p}_{\perp}^{\prime}\left(t^{\prime}\right)\right]$ passes through the phase-space point $\left(\mathbf{x}_{\perp}, \mathbf{p}_{\perp}\right)$ at time $t^{\prime}=t$ [1]. Here, $\theta^{\prime}\left(t^{\prime}=t\right)=\theta$, and the orbit $\theta^{\prime}\left(t^{\prime}\right)-\theta^{\prime}(t)$ is a function of the time difference $t^{\prime}-$ $t=\tau$. We express

$$
\theta^{\prime}\left(t^{\prime}\right)-\theta^{\prime}(t)=\int_{t}^{t^{\prime}} \frac{P_{\theta}}{m_{b}\left[r^{\prime}\left(t^{\prime \prime}\right)\right]^{2}} d t^{\prime \prime}
$$

where $P_{\theta}=r p_{\theta}=x p_{y}-y p_{x}=$ const is the canonical angular momentum of a beam particle moving in the axisymmetric equilibrium configuration. It is convenient to rewrite Eq. (6) as

$$
\theta^{\prime}\left(t^{\prime}\right)-\theta^{\prime}(t)=\theta^{\prime}\left(t^{\prime}-t_{\min }^{\prime}\right)-\theta^{\prime}\left(t-t_{\min }^{\prime}\right)
$$

where $t_{\min }^{\prime}$ is defined by $r^{\prime}\left(t_{\min }^{\prime}\right)=r_{\min }^{\prime}\left(H_{\perp}, P_{\theta}\right)$. Here, $r_{\min }^{\prime}\left(H_{\perp}, P_{\theta}\right)$ is the minimum radial excursion of the particle trajectory undergoing periodic motion. The functions $r^{\prime}\left(t^{\prime}\right)$ and $\theta^{\prime}\left(t^{\prime}-t_{\min }^{\prime}\right)-\omega_{\theta}\left(t^{\prime}-t_{\min }^{\prime}\right)$ are periodic functions with period $T_{r}=2 \pi / \omega_{r}$, where $\omega_{r}\left(H_{\perp}, P_{\theta}\right)$ is the frequency of radial oscillations, and $\omega_{\theta}\left(H_{\perp}, P_{\theta}\right)=$ $\Delta \theta / T_{r}$ is the average frequency of angular rotation. Here $\Delta \theta$ is the change in angle $\theta^{\prime}\left(t^{\prime}\right)$ during the period of time $T_{r}$. We expand in the Fourier series representation

$$
\begin{aligned}
& \delta \hat{\phi}\left[r^{\prime}\left(t^{\prime}\right)\right] \exp \left\{\operatorname{im}\left[\theta^{\prime}\left(t^{\prime}-t_{\min }^{\prime}\right)-\omega_{\theta}\left(t^{\prime}-t_{\min }^{\prime}\right)\right]\right\} \\
& \quad=\sum_{n} \exp \left[i n \omega_{r}\left(t^{\prime}-t_{\min }^{\prime}\right)\right] \int_{0}^{T_{r}} \frac{d \tau}{T_{r}} \hat{\delta} \hat{\phi}[\hat{r}(\tau)] \exp \left\{-i n \omega_{r} \tau+i m\left[\theta^{\prime}(\tau)-\omega_{\theta} \tau\right]\right\},
\end{aligned}
$$

where $\hat{r}(\tau)$ is defined by the equation

$$
\tau=\int_{r_{\min }^{\prime}}^{\hat{r}(\tau)} \frac{d \bar{r}}{\sqrt{2 m_{b}\left[H_{\perp}-P_{\theta}^{2} / 2 m_{b} \bar{r}^{2}-\psi_{0}(\bar{r})\right]}},
$$

and $\psi_{0}(\bar{r})=m_{b} \omega_{f}^{2} \bar{r}^{2} / 2+e_{b} \phi_{0}(\bar{r})$. Substituting Eq. (8) into Eq. (5) and integrating over $t^{\prime}$, we obtain

$$
\delta \hat{f}_{b}(r, \mathbf{p})=e_{b} \frac{\partial f_{b}^{0}}{\partial H_{\perp}} \hat{\boldsymbol{\phi} \phi}+e_{b}\left[\left(\omega-k_{z} v_{z}\right) \frac{\partial f_{b}^{0}}{\partial H_{\perp}}+k_{z} v_{z} \frac{\partial f_{b}^{0}}{\partial H_{\|}}\right] \sum_{n} \frac{\exp \left[i n \omega_{r} \hat{t}+i m \omega_{\theta} \hat{t}-i m \theta^{\prime}(\hat{t})\right]}{n \omega_{r}+m \omega_{\theta}+k_{z} v_{z}-\omega} I^{n, m}\left(H_{\perp}, P_{\theta}\right)
$$

In Eq. (10), the quantity $\hat{t}$ can be expressed as

$$
\hat{t}\left(r, H_{\perp}, P_{\theta}\right)=\int_{r_{\min }^{\prime}}^{r} \frac{d \bar{r}}{\sqrt{2 m_{b}\left[H_{\perp}-P_{\theta}^{2} / 2 m_{b} \bar{r}^{2}-\psi_{0}(\bar{r})\right.}},
$$

and $\theta^{\prime}(\hat{t})$ can be expressed as

$$
\theta^{\prime}(\hat{t})=\int_{r_{\min }^{\prime}}^{r} \frac{P_{\theta}}{m_{b} \bar{r}^{2}} \frac{\partial \hat{t}\left(\bar{r}, H_{\perp}, P_{\theta}\right)}{\partial \bar{r}} d \bar{r} .
$$

Furthermore, $I^{n, m}\left(H_{\perp}, P_{\theta}\right)$ is defined by

$$
\begin{aligned}
I^{n, m}\left(H_{\perp}, P_{\theta}\right)=\int_{0}^{T_{r}} \frac{d \tau}{T_{r}} \hat{\delta \phi}[\hat{r}(\tau)] \exp \{ & -i n \omega_{r} \tau \\
& \left.+i m\left[\theta^{\prime}(\tau)-\omega_{\theta} \tau\right]\right\} .
\end{aligned}
$$

In Eq. (4), we express the perturbation amplitude as $\hat{\delta \phi}(r)=\sum_{n} \alpha_{n} \phi_{n}(r)$, where $\left\{\alpha_{n}\right\}$ are constants, and the complete set of vacuum eigenfunctions $\left\{\phi_{n}(r)\right\}$ is defined by $\phi_{n}(r)=A_{n} J_{m}\left(\lambda_{n} r / r_{w}\right)$. Here, $\lambda_{n}$ is the $n$th zero of $J_{m}\left(\lambda_{n}\right)=0$, and $A_{n}=1 / r_{w}$ is a normalization constant such that $\int_{Q}^{r_{w}} d r r \phi_{n}(r) \phi_{n^{\prime}}(r)=(1 / 2) J_{m+1}^{2}\left(\lambda_{n}\right) \delta_{n, n^{\prime}}$. We substitute $\delta \phi(r)=\sum_{n} \alpha_{n} \phi_{n}(r)$ into Poisson's Eq. (4) and operate with $\int_{0}^{r_{w}} d r r \phi_{n^{\prime}}(r) \cdots$. This gives the matrix dispersion equation

$$
\sum_{n} \alpha_{n} D_{n, n^{\prime}}(\omega)=0
$$

where $D_{n, n^{\prime}}(\omega)$ is defined by

$$
D_{n, n^{\prime}}(\omega)=\frac{J_{m+1}^{2}\left(\lambda_{n}\right)}{2}\left(\lambda_{n}^{2}+k_{z}^{2} r_{w}^{2}\right) \delta_{n, n^{\prime}}+\chi_{n, n^{\prime}}(\omega)
$$

and the beam-induced susceptibility $\chi_{n, n^{\prime}}(\omega)$ is defined by

$$
\chi_{n, n^{\prime}}(\omega)=-4 \pi e_{b} r_{w}^{2} \int_{0}^{r_{w}} d r r \phi_{n^{\prime}}(r) \int d^{3} p \delta \hat{f}_{b}^{n}(r, \mathbf{p}) .
$$

Here, $\delta \hat{f}_{b}^{n}(r, \mathbf{p})$ is defined by Eq. (10) with $\hat{\delta \phi} \rightarrow \phi_{n}$.

By changing the integration variables in Eq. (16) from $\left\{r, p_{r}, p_{\theta}\right\}$ to $\left\{\hat{t}, H_{\perp}, P_{\theta}\right\}$, where $\hat{t}\left(r, H_{\perp}, P_{\theta}\right)$ is the time measured along the particle trajectory from the point where the radial distance is equal to $r_{\min }^{\prime}$ defined in Eq. (11), the integration volume transforms according to $r d r d p_{r} d p_{\theta}=d P_{\theta} d H_{\perp} d \hat{t}$. Using Eq. (10), Eq. (16) can be rewritten as 


$$
\begin{aligned}
\chi_{n, n^{\prime}}(\omega)= & \frac{r_{w}^{2}}{\lambda_{d}^{2}} q_{n, n^{\prime}} \\
& -\frac{1}{m_{b} \lambda_{d}^{2}} \sum \int \frac{d P_{\theta}}{\omega_{r}} \frac{d H_{\perp}}{T_{\perp b}} d p_{z} \exp \left[-\frac{H_{\perp}}{T_{\perp b}}\right] f_{M}\left(p_{z}\right)\left[\frac{\omega-k_{z} v_{z}}{\omega-p \omega_{r}-m \omega_{\theta}-k_{z} v_{z}}+\frac{k_{z}^{2} T_{\perp b} / m_{b}}{\left(\omega-p \omega_{r}-m \omega_{\theta}-k_{z} v_{z}\right)^{2}}\right]\left(I_{n}^{p, m}\right)^{*} I_{n^{\prime}}^{p, m}
\end{aligned}
$$

where $\lambda_{d}^{2}=T_{\perp b} / 4 \pi e_{b}^{2} \hat{n}_{b}$ is the perpendicular Debye length squared, and $f_{M}\left(p_{z}\right)=\left(2 \pi m_{b} T_{\| b}\right)^{-1 / 2} \times$ $\exp \left(-p_{z}^{2} / 2 m_{b} T_{\| b}\right)$. In Eq. (17), $q_{n, n^{\prime}}$ and $I_{n}^{p, m}$ are defined by

$$
q_{n, n^{\prime}}=\int_{0}^{1} d x x N\left(x r_{w}\right) J_{m}\left(\lambda_{n} x\right) J_{m}\left(\lambda_{n^{\prime}} x\right),
$$

and

$$
\begin{aligned}
I_{n}^{p, m}\left(H_{\perp}, P_{\theta}\right)=\int_{0}^{T_{r}} \frac{d \tau}{T_{r}} J_{m}\left[\frac{\lambda_{n} \hat{r}(\tau)}{r_{w}}\right] \exp \{ & -i p \omega_{r} \tau \\
& \left.+i m\left[\theta^{\prime}(\tau)-\omega_{\theta} \tau\right]\right\} .
\end{aligned}
$$

In Eq. (18), ( ()* denotes the complex conjugate, and $N\left(x r_{w}\right)=n_{b}^{0}\left(x r_{w}\right) / \hat{n}_{b}$ is the normalized density profile, where $n_{b}^{0}(r)=\int d^{3} p f_{b}^{0}(r, \mathbf{p})$. The condition for a nontrivial solution to Eq. (14) is

$$
\operatorname{det}\left\{D_{n, n^{\prime}}(\omega)\right\}=0,
$$

which plays the role of a matrix dispersion relation that determines the complex oscillation frequency $\omega$.

In the following analysis, it is convenient to introduce the effective depressed betatron frequency $\omega_{\beta \perp}$. It can be shown [1] that for the equilibrium distribution in Eq. (1), the mean-square beam radius $r_{b}^{2}$ defined by

$$
r_{b}^{2}=\left\langle r^{2}\right\rangle=\frac{\int d r r^{3} n_{b}^{0}(r)}{\int d r r n_{b}^{0}(r)}
$$

is related exactly to the line density $N_{b}=2 \pi \int d r r n_{b}^{0}(r)$ and transverse beam temperature $T_{\perp b}$ by the equilibrium radial force balance equation [1]

$$
\omega_{f}^{2} r_{b}^{2}=\frac{N_{b} e_{b}^{2}}{m_{b}}+\frac{2 T_{\perp b}}{m_{b}} .
$$

Equation (22) can be rewritten as

$$
\left(\omega_{f}^{2}-\frac{1}{2} \bar{\omega}_{p b}^{2}\right) r_{b}^{2}=\frac{2 T_{\perp b}}{m_{b}}
$$

where we have introduced the effective average beam plasma frequency $\bar{\omega}_{p b}$ defined by

$$
r_{b}^{2} \overline{\boldsymbol{\omega}}_{p b}^{2} \equiv \int_{0}^{r_{w}} d r r \omega_{p b}^{2}(r)=\frac{2 e_{b}^{2} N_{b}}{m_{b}} .
$$

Then, Eq. (23) can be used to introduce the effective depressed betatron frequency $\omega_{\beta \perp}$ defined by

$$
\omega_{\beta \perp}^{2} \equiv\left(\omega_{f}^{2}-\frac{1}{2} \bar{\omega}_{p b}^{2}\right)=\frac{2 T_{\perp b}}{m_{b} r_{b}^{2}} .
$$

If, for example, the beam density were uniform over the beam cross section, then Eq. (25) corresponds to the usual definition of the depressed betatron frequency for a $\mathrm{KV}$ beam, and it is readily shown that the radial orbit $\hat{r}(\tau)$ occurring in Eqs. (9), (13), and (19) can be expressed as

$$
\hat{r}^{2}(\tau)=\frac{H_{\perp}}{m_{b} \omega_{\beta \perp}^{2}}\left[1-\sqrt{1-\left(\frac{\omega_{\beta \perp} P_{\theta}}{H_{\perp}}\right)^{2}} \cos \left(2 \omega_{\beta \perp} \tau\right)\right] .
$$

In general, for the choice of equilibrium distribution function in Eq. (1), there will be a spread in transverse depressed betatron frequencies $\omega_{\beta \perp}\left(H_{\perp}, P_{\theta}\right)$, and the particle trajectories will not be described by the simple trigonometric function in Eq. (26). For present purposes, however, we consider a simple model in which the radial orbit $\hat{r}(\tau)$ occurring in Eqs. (9), (13), and (19) is approximated by Eq. (26) with the constant frequency $\omega_{\beta \perp}$ defined in Eq. (25), and the approximate equilibrium density profile is defined by $n_{b}^{0}(r)=$ $\hat{n}_{b} \exp \left(-m_{b} \omega_{\beta \perp}^{2} r^{2} / 2 T_{\perp b}\right)$. For a nonuniform beam, $\omega_{\beta \perp}^{-1}$ is the characteristic time for a particle with thermal speed $v_{\text {th } \perp}=\left(2 T_{\perp b} / m_{b}\right)^{1 / 2}$ to cross the rms radius $r_{b}$ of the beam. In this case, it is shown in Appendix B that $D_{n, n^{\prime}}(\omega)$ can be evaluated in closed analytical form provided the conducting wall is sufficiently far removed from the beam $\left(r_{w} / r_{b} \gtrless 3\right.$, say). In the limit of an anisotropic beam distribution that is cold in the longitudinal direction, i.e.,

$$
\frac{T_{\| b}}{T_{\perp b}} \rightarrow 0
$$

the result is

$$
\begin{aligned}
D_{n, n^{\prime}}^{m=2 l}= & \frac{J_{m+1}^{2}\left(\lambda_{n}\right)}{2}\left(\lambda_{n}^{2}+k_{z}^{2} r_{w}^{2}\right) \delta_{n, n^{\prime}}+\frac{\hat{\omega}_{p}^{2}}{\omega_{\beta \perp}^{2}} \exp \left(-z_{n, n}-z_{n^{\prime}, n^{\prime}}\right) \\
& \times\left[I_{2 l}\left(2 z_{n, n^{\prime}}\right)-I_{l}^{2}\left(z_{n, n^{\prime}}\right)\left(1+\frac{k_{z}^{2} v_{\mathrm{th} \perp}^{2}}{2 \omega^{2}}\right)-\sum_{q=1}^{\infty} I_{q-l}\left(z_{n, n^{\prime}}\right) I_{q+l}\left(z_{n, n^{\prime}}\right)\left(\frac{2 \omega^{2}}{\omega^{2}-4 q^{2} \omega_{\beta \perp}^{2}}+k_{z}^{2} v_{\mathrm{th} \perp}^{2} \frac{\omega^{2}+4 q^{2} \omega_{\beta \perp}^{2}}{\left(\omega^{2}-4 q^{2} \omega_{\beta \perp}^{2}\right)^{2}}\right)\right]
\end{aligned}
$$

for even values of $m=2 l$, and 


$$
\begin{aligned}
D_{n, n^{\prime}}^{m=2 l+1}= & \frac{J_{m+1}^{2}\left(\lambda_{n}\right)}{2}\left(\lambda_{n}^{2}+k_{z}^{2} r_{w}^{2}\right) \delta_{n, n^{\prime}}+\frac{\hat{\omega}_{p}^{2}}{\omega_{\beta \perp}^{2}} \exp \left(-z_{n, n}-z_{n^{\prime}, n^{\prime}}\right) \\
& \times\left[I_{2 l+1}\left(2 z_{n, n^{\prime}}\right)-\sum_{q=0}^{\infty} I_{q-l}\left(z_{n, n^{\prime}}\right) I_{q+l+1}\left(z_{n, n^{\prime}}\right)\left(\frac{2 \omega^{2}}{\omega^{2}-(2 q+1)^{2} \omega_{\beta \perp}^{2}}+k_{z}^{2} v_{\mathrm{th} \perp}^{2} \frac{\omega^{2}+(2 q+1)^{2} \omega_{\beta \perp}^{2}}{\left[\omega^{2}-(2 q+1)^{2} \omega_{\beta \perp}^{2}\right]^{2}}\right)\right]
\end{aligned}
$$

for odd values of $m=2 l+1$. Here, $z_{n, n^{\prime}}=\left(r_{b} / r_{w}\right)^{2} \lambda_{n} \lambda_{n^{\prime}} / 4$. In this case, the matrix elements decrease exponentially away from the diagonal, with

$$
\left|\frac{D_{n, n+k}}{D_{n, n}}\right| \sim \exp \left(-\frac{\pi^{2} k^{2}}{4} \frac{r_{b}^{2}}{r_{w}^{2}}\right)
$$

where $k$ is an integer, and we have used the approximate relation $\lambda_{n} \approx \pi(4 n+2 m-1) / 4$. Therefore, for $r_{w} / r_{b} \gtrsim 3$, we can approximate $\left\{D_{n, n^{\prime}}(\omega)\right\}$ by a tridiagonal matrix. In this case, for the lowest-order radial modes $(n=1$ and $n=2)$, the matrix dispersion relation (20) can be approximated by

$$
D_{1,1}(\omega) D_{2,2}(\omega)-\left[D_{1,2}(\omega)\right]^{2}=0
$$

where use has been made of $D_{1,2}(\omega)=D_{2,1}(\omega)$.

We introduce the effective perpendicular thermal speed squared of a beam particle defined by $v_{\text {th } \perp}^{2}=2 T_{\perp b} / m_{b}$. Then, for $T_{\| b} / T_{\perp b} \rightarrow 0$ and $r_{w} / r_{b} \gtrsim 3$, the approximate dispersion relation (31) describing the coupling of the lowestorder $n=1$ mode with the $n=2$ radial mode, within the context of the present simplified model, can be expressed as

$$
\begin{aligned}
& \left\{\lambda_{1}^{2}+k_{z}^{2} r_{w}^{2}+\frac{2 \exp \left(-\frac{k_{1}^{2}}{2}\right)\left(\hat{\omega}_{p}^{2} / \omega_{\beta \perp}^{2}\right)}{J_{m+1}^{2}\left(\lambda_{1}\right)}\left[I_{m}\left(\frac{k_{1}^{2}}{2}\right)-\left(1+\frac{k_{z}^{2} v_{\mathrm{th} \perp}^{2}}{2 \omega^{2}}\right) I_{m / 2}^{2}\left(\frac{k_{1}^{2}}{4}\right)-\left(\frac{2 \omega^{2}}{\omega^{2}-4 \omega_{\beta \perp}^{2}}+\frac{k_{z}^{2} v_{\mathrm{th} \perp}^{2}\left(\omega^{2}+4 \omega_{\beta \perp}^{2}\right)}{\left(\omega^{2}-4 \omega_{\beta \perp}^{2}\right)^{2}}\right) I_{m / 2+1}\left(\frac{k_{1}^{2}}{4}\right) I_{m / 2-1}\left(\frac{k_{1}^{2}}{4}\right)\right]\right\} \\
& \left\{\lambda_{2}^{2}+k_{z}^{2} r_{w}^{2}+\frac{2 \exp \left(-\frac{k_{2}^{2}}{2}\right)\left(\hat{\omega}_{p}^{2} / \omega_{\beta \perp}^{2}\right)}{J_{m+1}^{2}\left(\lambda_{2}\right)}\left[I_{m}\left(\frac{k_{2}^{2}}{2}\right)-\left(1+\frac{k_{z}^{2} v_{\mathrm{th} \perp}^{2}}{2 \omega^{2}}\right) I_{m / 2}^{2}\left(\frac{k_{2}^{2}}{4}\right)-\left(\frac{2 \omega^{2}}{\omega^{2}-4 \omega_{\beta \perp}^{2}}+\frac{k_{z}^{2} v_{\mathrm{th} \perp}^{2}\left(\omega^{2}+4 \omega_{\beta \perp}^{2}\right)}{\left(\omega^{2}-4 \omega_{\beta \perp}^{2}\right)^{2}}\right) I_{m / 2+1}\left(\frac{k_{2}^{2}}{4}\right) I_{m / 2-1}\left(\frac{k_{2}^{2}}{4}\right)\right]\right\} \\
& =\frac{\left(2 \hat{\omega}_{p}^{2} / \omega_{\beta \perp}^{2}\right)^{2}}{J_{m+1}^{2}\left(\lambda_{1}\right) J_{m+1}^{2}\left(\lambda_{2}\right)} \exp \left(-\frac{\left(k_{1}^{2}+k_{2}^{2}\right)}{2}\right)\left\{I_{m}\left(\frac{k_{1} k_{2}}{2}\right)-\left(1+\frac{k_{z}^{2} v_{\mathrm{th} \perp}^{2}}{2 \omega^{2}}\right) I_{m / 2}^{2}\left(\frac{k_{1} k_{2}}{4}\right)\right. \\
& \left.-\left(\frac{2 \omega^{2}}{\omega^{2}-4 \omega_{\beta \perp}^{2}}+\frac{k_{z}^{2} v_{\mathrm{th} \perp}^{2}\left(\omega^{2}+4 \omega_{\beta \perp}^{2}\right)}{\left(\omega^{2}-4 \omega_{\beta \perp}^{2}\right)^{2}}\right) I_{m / 2+1}\left(\frac{k_{1} k_{2}}{4}\right) I_{m / 2-1}\left(\frac{k_{1} k_{2}}{4}\right)\right\}^{2},
\end{aligned}
$$

for even values of azimuthal mode numbers $m=0,2,4, \ldots$, and by

$$
\begin{aligned}
& \left\{\lambda_{1}^{2}+k_{z}^{2} r_{w}^{2}+\frac{2 \exp \left(-\frac{k_{1}^{2}}{2}\right)\left(\hat{\omega}_{p}^{2} / \omega_{\beta \perp}^{2}\right)}{J_{m+1}^{2}\left(\lambda_{1}\right)}\left[I_{m}\left(\frac{k_{1}^{2}}{2}\right)-\left(\frac{2 \omega^{2}}{\omega^{2}-\omega_{\beta \perp}^{2}}+\frac{k_{z}^{2} v_{\mathrm{th \perp}}^{2}\left(\omega^{2}+\omega_{\beta \perp}^{2}\right)}{\left(\omega^{2}-\omega_{\beta \perp}^{2}\right)^{2}}\right) I_{(m+1) / 2}\left(\frac{k_{1}^{2}}{4}\right) I_{(m-1) / 2}\left(\frac{k_{1}^{2}}{4}\right)\right]\right\} \\
& \left\{\lambda_{2}^{2}+k_{z}^{2} r_{w}^{2}+\frac{2 \exp \left(-\frac{k_{2}^{2}}{2}\right)\left(\hat{\omega}_{p}^{2} / \omega_{\beta \perp}^{2}\right)}{J_{m+1}^{2}\left(\lambda_{2}\right)}\left[I_{m}\left(\frac{k_{2}^{2}}{2}\right)-\left(\frac{2 \omega^{2}}{\omega^{2}-\omega_{\beta \perp}^{2}}+\frac{k_{z}^{2} v_{\mathrm{th \perp}}^{2}\left(\omega^{2}+\omega_{\beta \perp}^{2}\right)}{\left(\omega^{2}-\omega_{\beta \perp}^{2}\right)^{2}}\right) I_{(m+1) / 2}\left(\frac{k_{2}^{2}}{4}\right) I_{(m-1) / 2}\left(\frac{k_{2}^{2}}{4}\right)\right]\right\} \\
& =\frac{\left(2 \hat{\omega}_{p}^{2} / \omega_{\beta \perp}^{2}\right)^{2}}{J_{m+1}^{2}\left(\lambda_{1}\right) J_{m+1}^{2}\left(\lambda_{2}\right)} \exp \left(-\frac{\left(k_{1}^{2}+k_{2}^{2}\right)}{2}\right)\left\{I_{m}\left(\frac{k_{1} k_{2}}{2}\right)-\left(\frac{2 \omega^{2}}{\omega^{2}-\omega_{\beta \perp}^{2}}+\frac{k_{z}^{2} v_{\mathrm{th} \perp}^{2}\left(\omega^{2}+\omega_{\beta \perp}^{2}\right)}{\left(\omega^{2}-\omega_{\beta \perp}^{2}\right)^{2}}\right) I_{(m+1) / 2}\left(\frac{k_{1} k_{2}}{4}\right) I_{(m-1) / 2}\left(\frac{k_{1} k_{2}}{4}\right)\right\}^{2},
\end{aligned}
$$

for odd values of $m=1,3,5, \ldots$. In Eqs. (32) and (33), we have retained only the leading-order nonresonant terms and one resonant term at frequencies $\omega \approx \pm 2 \omega_{\beta \perp}$ for even values of $m$, and $\omega \approx \pm \omega_{\beta \perp}$ for odd values of $m$. In the dispersion relations (32) and (33), $\lambda_{1}$ and $\lambda_{2}$ are determined from the zeros of $J_{m}\left(\lambda_{n}\right)=0$, the quantity $v_{\text {th } \perp}=\left(2 T_{\perp b} / m_{b}\right)^{1 / 2}$ is the transverse thermal speed, $k_{1}$ and $k_{2}$ are defined by $k_{1}=\lambda_{1} r_{b} / r_{w}$ and $k_{2}=\lambda_{2} r_{b} / r_{w}$, and $\omega_{\beta \perp}=\omega_{f}\left(1-\bar{s}_{b}\right)^{1 / 2}$ is the effective depressed betatron frequency [Eq. (25)], where $\bar{s}_{b}=\bar{\omega}_{p b}^{2} / 2 \omega_{f}^{2}$ is the effective normalized beam intensity defined in terms of $\overline{\boldsymbol{\omega}}_{p b}$.
The dispersion relations (32) and (33) can be used to investigate detailed electrostatic stability properties for strong anisotropy $\left(T_{\| b} / T_{\perp b} \rightarrow 0\right)$ for a wide range of normalized axial wave numbers $\left(k_{z} r_{w}\right)$ and effective normalized beam intensity $\bar{s}_{b}=\bar{\omega}_{p b}^{2} / 2 \omega_{f}^{2}$, or equivalently, normalized tune depression $\bar{\nu} / \nu_{0}$ defined by

$$
\frac{\bar{\nu}}{\nu_{0}} \equiv \frac{\omega_{\beta \perp}}{\omega_{f}}=\left(1-\bar{s}_{b}\right)^{1 / 2} .
$$

For sufficiently large values of $k_{z} r_{w}$, the large temperature anisotropy $\left(T_{\| b} / T_{\perp b} \rightarrow 0\right)$ in Eqs. (32) and (33) provides 
the free energy to drive the classical Harris-type instability [26,27], generalized here to include finite transverse geometry and beam space-charge effects. The influence of the finite longitudinal temperature can be taken into account if one assumes $T_{\| b} \neq 0$ in Eq. (1). This results in the (collisionless) Landau damping of the unstable mode due to resonant wave-particle interactions [1] associated with the axial momentum spread of the beam particles. The dispersion relation for the case of nonzero longitudinal temperature $T_{\| b} \neq 0$ is derived in Appendix B.

To compare with the simulation results in Sec. IV, we introduce the normalized on-axis beam intensity $s_{b}$ defined in terms of the on-axis $(r=0)$ beam density $\hat{n}_{b}$. Here, $s_{b} \equiv \hat{\omega}_{p b}^{2} / 2 \omega_{f}^{2}$, where $\hat{\omega}_{p b}=\left(4 \pi e_{b}^{2} \hat{n}_{b} / m_{b}\right)^{1 / 2}$. Using Eqs. (22) -(25), the average normalized beam intensity $\bar{s}_{b}=\bar{\omega}_{p b}^{2} / 2 \omega_{f}^{2}$ introduced in Eq. (34) is related to $s_{b}$ by the equation

$$
s_{b}=\bar{s}_{b} \hat{n}_{b}\left(\int_{0}^{r_{w}} d r r^{3} n_{b}^{0}(r)\right) /\left(\int_{0}^{r_{w}} d r r n_{b}^{0}(r)\right)^{2} .
$$

The allowed range of the normalized intensity parameter $s_{b}$ is $0 \leq s_{b}<1$, where the limit $s_{b} \rightarrow 1$ corresponds to a fully depressed tune (space-charge-dominated limit).

Typical numerical results obtained from the approximate dispersion relation utilizing Eqs. (B9)-(B11) are
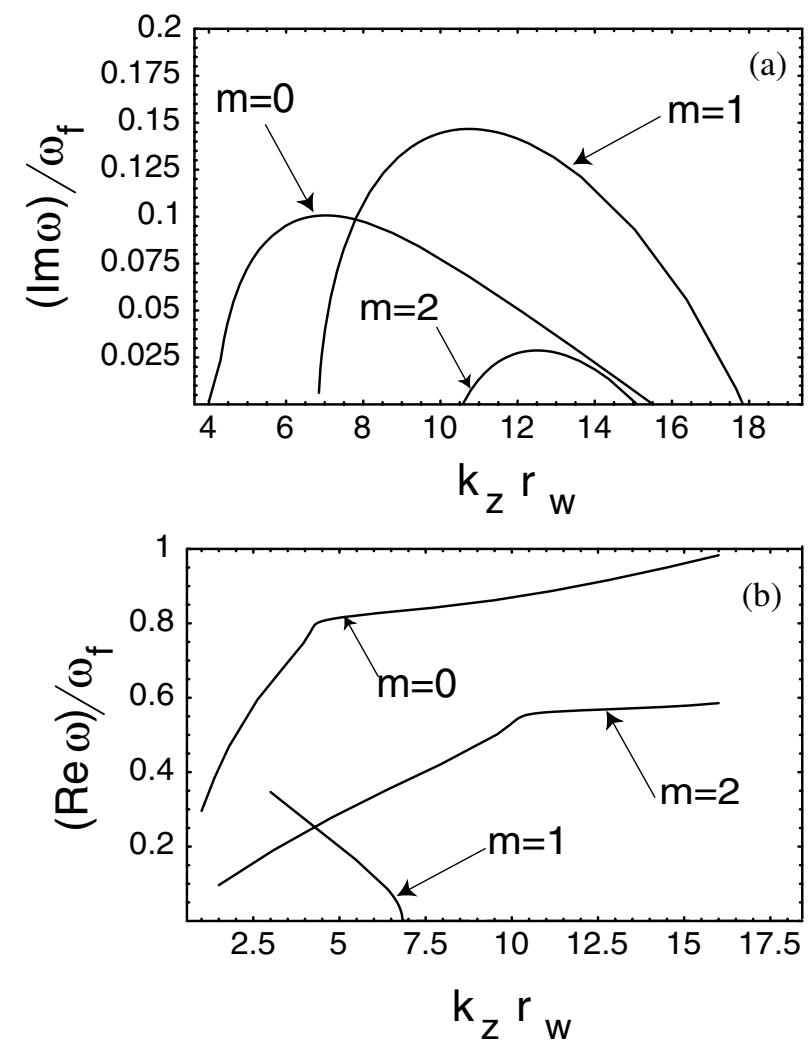

FIG. 1. Plots of normalized growth rate $(\operatorname{Im} \omega) / \omega_{f}$ and real frequency $(\operatorname{Re} \omega) / \omega_{f}$ versus $k_{z} r_{w}$ for $s_{b}=0.95$ and $T_{\| b} / T_{\perp b}=$ 0.02 . presented in Figs. 1-5 for the case where $r_{w}=3 r_{b}$. Only the leading-order nonresonant terms and one resonant term at frequencies $\omega \approx \pm 2 \omega_{\beta \perp}$ for even values of $m$, and $\omega \approx \pm \omega_{\beta \perp}$ for odd values of $m$, have been retained in the analysis. Figure 1 shows the normalized growth rate $(\operatorname{Im} \omega) / \omega_{f}$ and real frequency $(\operatorname{Re} \omega) / \omega_{f}$ plotted versus normalized wave number $k_{z} r_{w}$ for normalized on-axis beam intensity $s_{b}=0.95$ and temperature ratio $T_{\| b} / T_{\perp b}=0.02$. Note from Fig. 1 that the critical values of $k_{z} r_{w}$ for the onset of instability and for maximum growth rate increase as the azimuthal mode number $m$ is increased. As expected, finite $T_{\| b}$ effects introduce a finite bandwidth in $k_{z} r_{w}$ for instability, since the modes with large values of $k_{z} r_{w}$ are stabilized by Landau damping. Also, the unstable modes with odd azimuthal number are purely growing.

Figures 2 and 3 show plots of the normalized growth rate $(\operatorname{Im} \omega) / \omega_{f}$ and real frequency $(\operatorname{Re} \omega) / \omega_{f}$ at maximum growth versus normalized beam intensity $s_{b}$ (Fig. 2) and effective tune depression $\bar{\nu} / \nu_{0}$ (Fig. 3). The $m=1$ dipole mode has the highest growth rate, $(\operatorname{Im} \omega) / \omega_{f} \simeq$ 0.15 , for $\bar{\nu} / \nu_{0} \simeq 0.45$. Note from Fig. 2 that the critical value of $s_{b}$ for the onset of the instability and the value of $s_{b}^{\max }$ with maximum growth rate increase with azimuthal mode number $m$. The instability is absent for $s_{b}<0.65$ or $\bar{\nu} / \nu_{0}>0.77$ for the choice of parameters in Figs. 2 and 3.
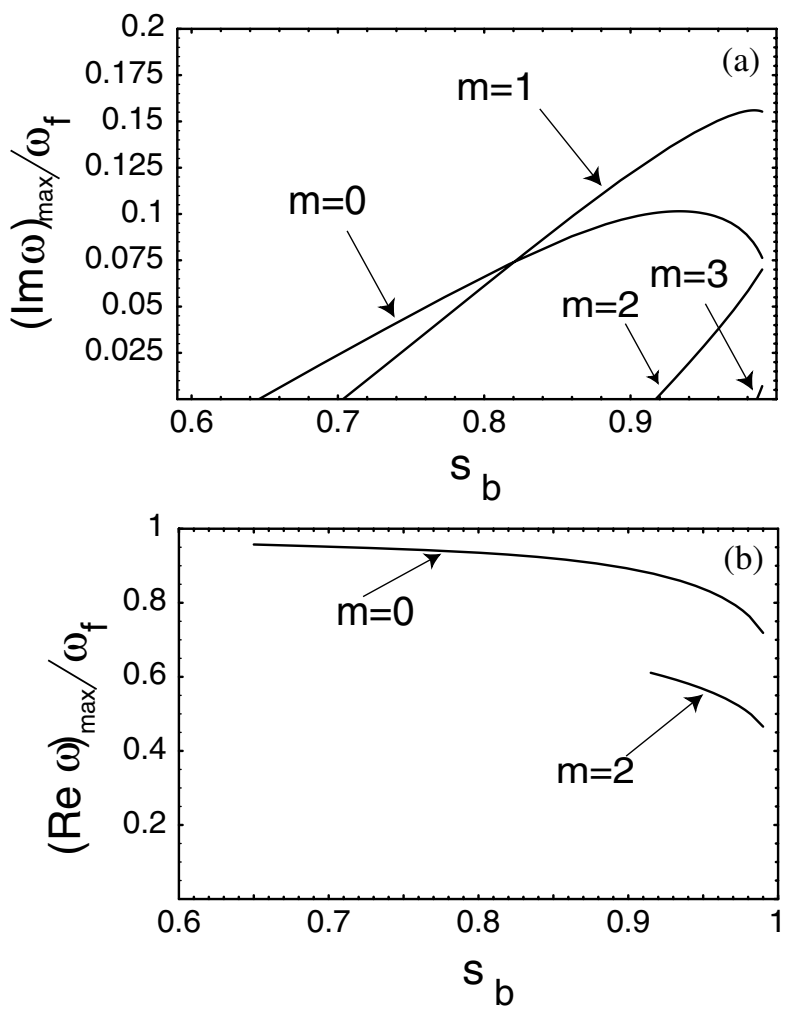

FIG. 2. Plots of normalized growth rate $(\operatorname{Im} \omega)_{\max } / \omega_{f}$ and real frequency $(\operatorname{Re} \omega)_{\max } / \omega_{f}$ at maximum growth rate versus normalized beam intensity $s_{b}$ for $T_{\| b} / T_{\perp b}=0.02$. 

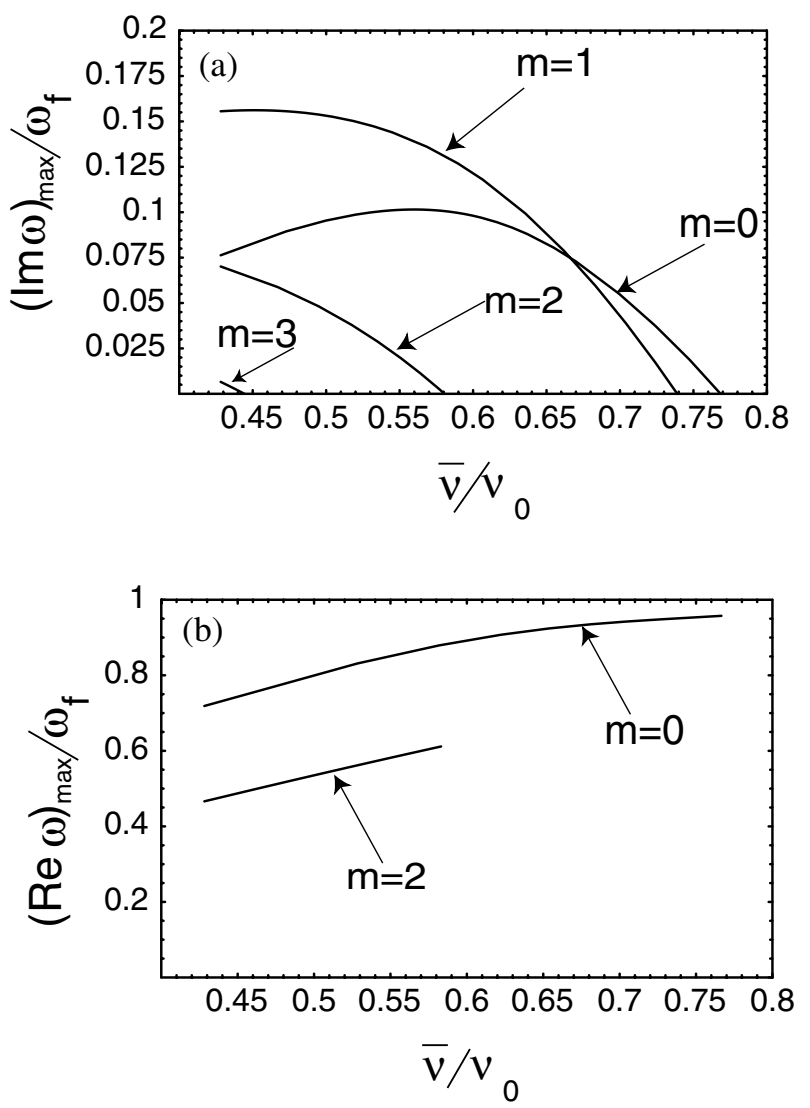

FIG. 3. Plots of normalized growth rate $(\operatorname{Im} \omega)_{\max } / \omega_{f}$ and real frequency $(\operatorname{Re} \omega)_{\max } / \omega_{f}$ at maximum growth versus normalized tune depression $\bar{\nu} / \nu_{0}$ for $T_{\| b} / T_{\perp b}=0.02$.

The real frequency $(\operatorname{Re} \omega) / \omega_{f}$ of the unstable modes with odd azimuthal numbers $m=1,3, \ldots$ are zero and are not plotted in Figs. 2 and 3. Moreover, the real frequency is plotted only for the unstable modes. The normalized eigenfunctions $\operatorname{Re} \delta \hat{\phi}(r)$ and $\operatorname{Im} \delta \hat{\phi}(r)$ corresponding to $s_{b}=0.95$ and maximum growth rate are plotted versus $r / r_{w}$ in Fig. 4. The real part of the eigenfunction for $m=$ 0 has no zeros and has a structure similar to the familiar longitudinal mode $\left(L_{1}\right)$ in Ref. [8].

An important characteristic of the instability, the longitudinal threshold temperature $T_{\| b}^{\text {th }}$ for the onset of instability normalized to the transverse temperature $T_{\perp b}$, is plotted in Fig. 5 versus normalized beam intensity $s_{b}$ and effective tune depression $\bar{\nu} / \nu_{0}$. The threshold temperature $T_{\| b}^{\text {th }}$ is a decreasing function of azimuthal wave number $m$ and normalized tune depression $\bar{\nu} / \nu_{0}$, and an increasing function of the normalized beam intensity $s_{b}$. Note from Fig. 5 that the instability is absent for $T_{\| b} / T_{\perp b}>0.22$.

\section{DESCRIPTION OF THE NONLINEAR $\delta f$ SIMULATION CODE}

The theoretical model described in Sec. II uses simplified assumptions for the particle trajectories necessary
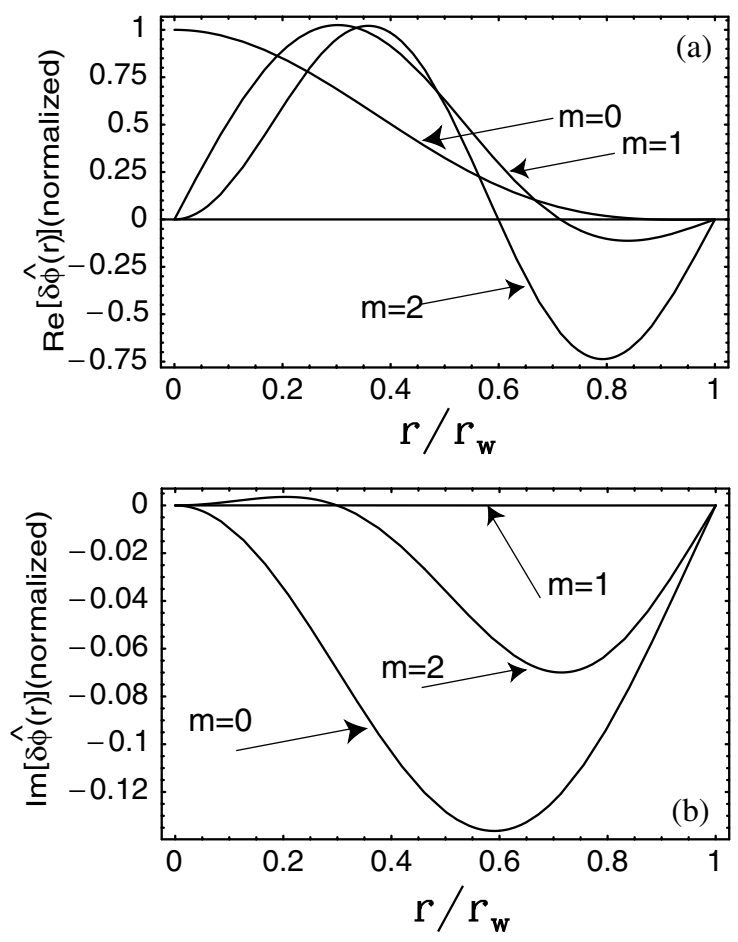

FIG. 4. Plots of normalized eigenfunctions $\operatorname{Re}[\delta \hat{\phi}(r)]$ and $\operatorname{Im}[\delta \hat{\phi}(r)]$ at maximum growth versus $r / r_{w}$ corresponding to $s_{b}=0.95$ and $T_{\| b} / T_{\perp b}=0.02$.
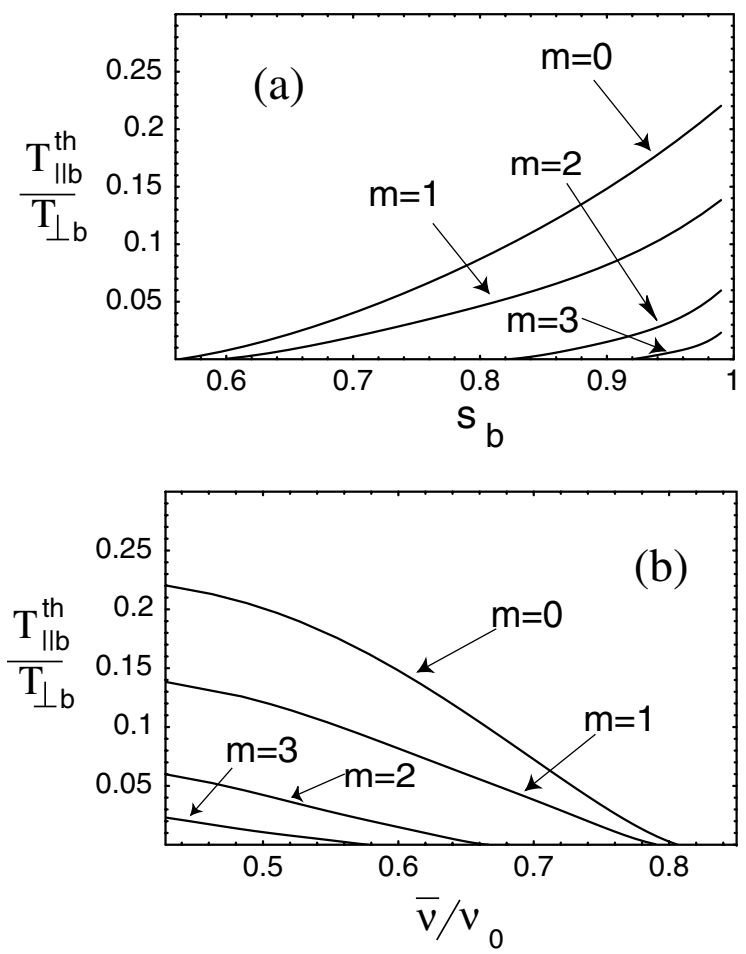

FIG. 5. Longitudinal threshold temperature $T_{\| b}^{\text {th }}$ for the onset of instability normalized to the transverse temperature $T_{\perp b}$ is plotted versus (a) normalized beam intensity $s_{b}$ and (b) normalized tune depression $\bar{\nu} / \nu_{0}$. 
to derive the dispersion matrix. This allowed us to obtain a closed expression for the dispersion matrix and to study the stability properties of intense beams with temperature anisotropy. To improve on this method, one can use Eqs. (17)-(19), together with the equations for the particle trajectories [Eqs. (9) and (12)] calculated numerically using the equilibrium space-charge potential obtained from Poisson's equation (2). The dispersion matrix obtained in this way can be used to determine the unstable mode frequencies and growth rates by numerically searching for the solutions to the dispersion equation (20) for the complex frequency $\omega=(\operatorname{Re} \omega, \operatorname{Im} \omega)$. Although such spectral method calculations are possible, they are less efficient than particle-in-cell (PIC) calculations. To investigate the stability properties numerically, we make use of the nonlinear $\delta f$ method [37] described below, as implemented in the BEST code $[1,15,17]$.

For present purposes, we make use of the smoothfocusing approximation in which the transverse applied focusing force is modeled by $\mathbf{F}_{\text {foc }}=-\gamma_{b} m_{b} \omega_{f}^{2} \mathbf{x}_{\perp}$, where $\omega_{f}$ is the constant focusing frequency associated with the applied focusing field, $m_{b}$ is the particle rest mass, $\gamma_{b}=$ $\left(1-\beta_{b}^{2}\right)^{1 / 2}$ is the relativistic mass factor, $V_{b}=\beta_{b} c=$ const is the average axial beam velocity, and $c$ is the speed of light in vacuo. In the $\delta f$ approach, the solutions to the nonlinear Vlasov-Maxwell equations are expressed as $f_{b}=f_{b}^{0}+\delta f_{b}, \phi=\phi^{0}+\delta \phi$, and $A_{z}=A_{z}^{0}+\delta A_{z}$, where $\left(f_{b}^{0}, \phi^{0}, A_{z}^{0}\right)$ are known equilibrium solutions $(\partial / \partial t=0)$. In the electromagnetostatic, approximation the self-fields are represented as $\mathbf{E}^{s}=-\nabla \phi(\mathbf{x}, t)$ and $\mathbf{B}^{s}=\nabla \times A_{z}(\mathbf{x}, t) \mathbf{e}_{z}$ [38]. The perturbed potentials satisfy the equations [15]

$$
\begin{gathered}
\nabla^{2} \delta \phi=-4 \pi e_{b} \int d^{3} p \delta f_{b}, \\
\nabla^{2} \delta A_{z}=-\frac{4 \pi}{c} e_{b} \int d^{3} p v_{z} \delta f_{b},
\end{gathered}
$$

where $e_{b}$ is the particle charge, and $\delta f_{b}(\mathbf{x}, \mathbf{p}, t)$ is given by the weighted Klimontovich representation,

$$
\delta f_{b}=\frac{N_{b}}{N_{s b}} \sum_{i=1}^{N_{s b}} w_{b i} \delta\left(\mathbf{x}-\mathbf{x}_{b i}\right) \delta\left(\mathbf{p}-\mathbf{p}_{b i}\right) .
$$

Here, $N_{s b}$ is the total number of beam simulation particles, $N_{b}$ is the total number of actual beam particles, and the weight function is defined by $w_{b} \equiv \delta f_{b} / f_{b}$. The nonlinear particle simulations are carried out by iteratively advancing the particle motion, including the weights they carry, according to [15]

$$
\begin{gathered}
\frac{d \mathbf{x}_{\perp b i}}{d t}=\left(\gamma_{b} m_{b}\right)^{-1} \mathbf{p}_{\perp b i}, \\
\frac{d z_{b i}}{d t}=v_{z b i}=\beta_{b} c+\gamma_{b}^{-3} m_{b}^{-1}\left(p_{z b i}-\gamma_{b} m_{b} \beta_{b} c\right)
\end{gathered}
$$

$$
\begin{gathered}
\frac{d \mathbf{p}_{b i}}{d t}=-\gamma_{b} m_{b} \omega_{f}^{2} \mathbf{x}_{\perp_{b i}}-e_{b}\left(\nabla \phi-\frac{v_{z b i}}{c} \nabla_{\perp} A_{z}\right), \\
\frac{d w_{b i}}{d t}=-\left(1-w_{b i}\right) \frac{1}{f_{b 0}} \frac{\partial f_{b 0}}{\partial \mathbf{p}} \cdot \delta\left(\frac{d \mathbf{p}_{b i}}{d t}\right), \\
\delta\left(\frac{d \mathbf{p}_{b i}}{d t}\right)=-e_{b}\left(\nabla \delta \phi-\frac{v_{z b i}}{c} \nabla_{\perp} \delta A_{z}\right),
\end{gathered}
$$

and updating the fields by solving the perturbed Maxwell's equations with appropriate boundary conditions at the cylindrical, perfectly conducting wall at radius $r_{w}$.

The $\delta f$ approach is fully equivalent to the original nonlinear Vlasov-Maxwell equations, but the noise associated with representation of the background distribution $f_{b}^{0}$ in conventional PIC simulations is removed. In the $\delta f$ approach, the simulation particles are used to represent only a small part of the entire distribution $\delta f_{b}=f_{b}$ $f_{b}^{0}$, and therefore the statistical error in the simulations is proportional to $\epsilon_{\delta f} \sim \delta f_{b} / \sqrt{N_{s b}}$, whereas the error in PIC simulations is proportional to $\epsilon_{\text {pic }} \sim f_{b} / \sqrt{N_{s b}}$. Therefore, the typical gain in accuracy in $\delta f$ simulations compared to PIC simulations with the same number of particles is $\epsilon_{\delta f} / \epsilon_{\text {pic }}=\bar{w}_{b i}$ [15]. This fact allows much more accurate simulations of the nonlinear dynamics and instability thresholds when $\left|\bar{w}_{b i}\right| \ll 1$. When the perturbation $\delta f_{b}$ becomes comparable in magnitude with the background distribution function $f_{b}^{0}$, then the $\delta f$ method becomes less accurate than a full PIC simulation. In the present paper, a hybrid combination of the $\delta f$ and PIC simulation methods is used, where the number density is calculated according to $\delta n_{b}=\left[1-\theta\left(\bar{w}_{b i}\right)\right] \delta n_{\delta f}+$ $\theta\left(\bar{w}_{b i}\right)\left(n_{\text {pic }}-n_{0}\right)$, where $\theta(w)$ is a monotonic function of its argument such that $\theta(w \rightarrow 0) \rightarrow 0$ and $\theta(w \rightarrow 1) \rightarrow 1$. Here, $\delta n_{\delta f}=\int d^{3} p \delta f_{b}$ and $n_{\text {pic }}=\int d^{3} p f_{b}$.

In addition, the $\delta f$ method can be used to study linear stability properties, provided all nonlinear terms in the dynamical equations (39)-(43) are neglected [15,17]. This corresponds to replacing the term $1-w_{b i}$ with 1 in Eq. (42) for the weights and moving particles along the trajectories calculated in the unperturbed potentials $\left(\phi^{0}, A_{z}^{0}\right)$.

The $\delta f$ method described above has been implemented in the three-dimensional electromagnetostatic particlein-cell code (BEST) in cylindrical geometry with a perfectly conducting cylindrical wall at radius $r_{w}$. Maxwell's equations (36) and (37) are solved using fast Fourier transform techniques in the longitudinal and azimuthal directions. The particle positions [Eqs. (39) and (41)] and weights [Eq. (42)] are advanced using a second-order predictor-corrector algorithm. The BEST code is parallelized using the Message Passing Interface (MPI) with domain decomposition in the direction of beam propagation. The NetCDF data format is used for large-scale 
diagnostics and visualization. Typical simulation runs consist of $10^{6}$ simulation particles and are performed on the IBM SP/RS 6000 at NERSC.

\section{SIMULATION RESULTS}

In this section we present the simulation results carried out in the beam frame $\left(V_{b}=0\right.$ and $\left.\gamma_{b}=1\right)$ for a continuous, anisotropic beam in a constant focusing field. The self-consistent equilibrium distribution function $(\partial / \partial t=0)$ in the beam frame is taken to be of the form given in Eq. (1), where $\hat{n}_{b}$ is the beam density at $r=0$, and $T_{\perp b}$ and $T_{\| b}$ are the transverse and longitudinal temperatures. The equilibrium self-field potentials $\left(\varphi_{0}, A_{z 0}\right)$ are determined numerically from Maxwell's equations [15]. It is also assumed that the beam is located inside a grounded, perfectly conducting cylindrical wall at radius $r_{w}=3 r_{b}$, where $r_{b}=\left[\left\langle r^{2}\right\rangle\right]^{1 / 2}$ is the rms beam radius. Random initial perturbations are introduced to the particle weights, and the beam is propagated from $t=0$ to $t=200 \omega_{f}^{-1}$. The initial temperature ratio is taken to be $T_{\| b} / T_{\perp b}=0.02$, and the simulations are performed in the beam frame with $V_{b}=0$ and $\gamma_{b}=1$. Typical numerical results are illustrated in Figs. 6-12, where the simulations have been carried out over a wide range of normalized beam intensities $s_{b}=\omega_{p b}^{2} / 2 \omega_{f}^{2}$ ranging from $s_{b}=0.2$ to $s_{b}=0.98$.

Using the linearized version of the 3D BEST code, Figs. 6-9 show results of the $\delta f$ simulations for perturbations with axial and azimuthal spatial dependence proportional to $\exp \left(i k_{z} z+i m \theta\right)$, where $k_{z}$ is the axial wave number, and $m$ is the azimuthal mode number. Figure 6 shows plots of the real and imaginary parts of the complex oscillation frequency $\omega$ versus normalized axial wave number $k_{z} r_{w}$, for $s_{b}=0.95$ and azimuthal mode numbers $m=0,1,2,3$. Note that the instability has a finite bandwidth with maximum growth rate occurring at $k_{z} r_{w} \simeq 9$. The dependence of the maximum growth rate $(\operatorname{Im} \omega)_{\max } / \omega_{f}$ and the normalized real frequency $(\operatorname{Re} \omega)_{\max } / \omega_{f}$ at maximum growth on normalized beam intensity $s_{b}$ and tune depression $\bar{\nu} / \nu_{0}$ are shown in Figs. 7 and 8 , respectively. Evidently, the maximum growth rate $(\operatorname{Im} \omega)_{\max } / \omega_{f}$ is an increasing function of beam intensity $s_{b}$ and a decreasing function of normalized tune depression $\bar{\nu} / \nu_{0}$. Note from Fig. 7 that the critical value of $s_{b}$ for the onset of the instability increases with azimuthal mode number $m$. The real frequency $(\operatorname{Re} \omega) / \omega_{f}$ of the unstable modes for odd azimuthal numbers $m=1,3$ is zero and is not plotted. The real frequency is plotted only for the unstable modes. Consistent with the analytical predictions in Sec. II, note that the dipole mode $(m=1)$ has the largest growth rate. Furthermore, all modes are found to be stable in the region $s_{b} \leq 0.5$, or equivalently $\bar{\nu} / \nu_{0} \geq 0.85$. The radial dependence of the eigenfunctions for the perturbed electrostatic potential obtained from the linearized BEST code at maximum
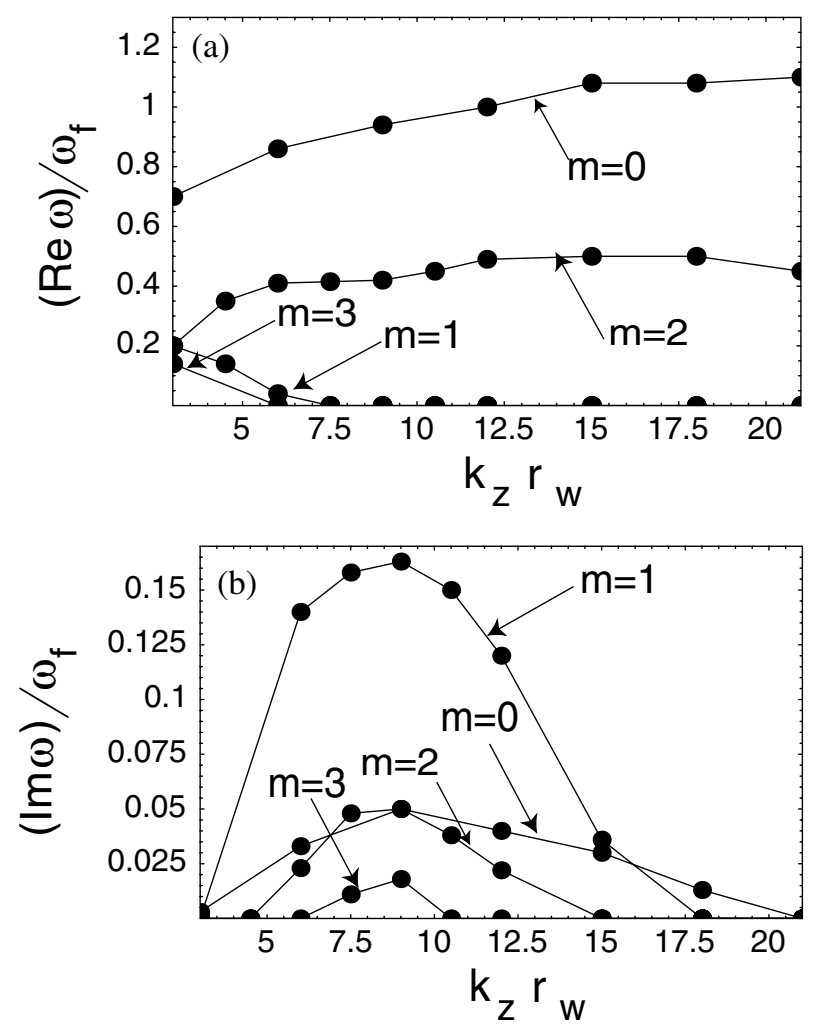

FIG. 6. Plots of normalized real frequency $(\operatorname{Re} \omega) / \omega_{f}$ and growth rate $(\operatorname{Im} \omega) / \omega_{f}$ versus $k_{z} r_{w}$ for $s_{b}=0.95$ and $T_{\| b} / T_{\perp b}=0.02$.
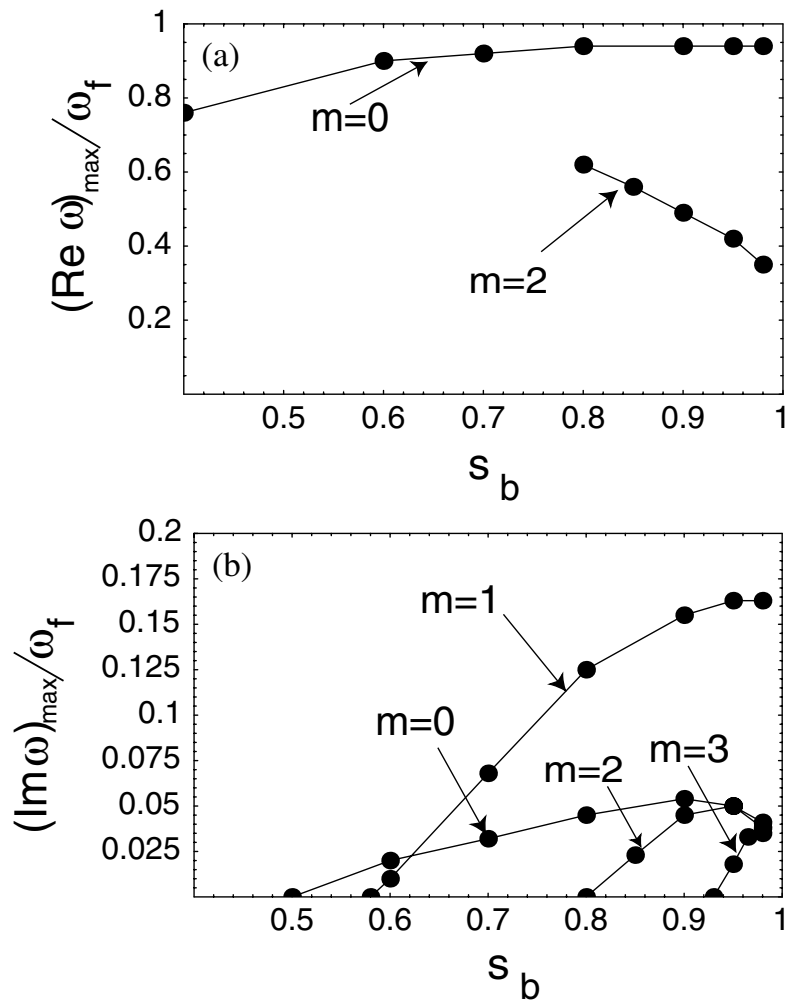

FIG. 7. Plots of normalized real frequency $(\operatorname{Re} \omega)_{\max } / \omega_{f}$ and growth rate $(\operatorname{Im} \omega)_{\max } / \omega_{f}$ at maximum growth versus normalized beam intensity $s_{b}$ for $T_{\| b} / T_{\perp b}=0.02$. 

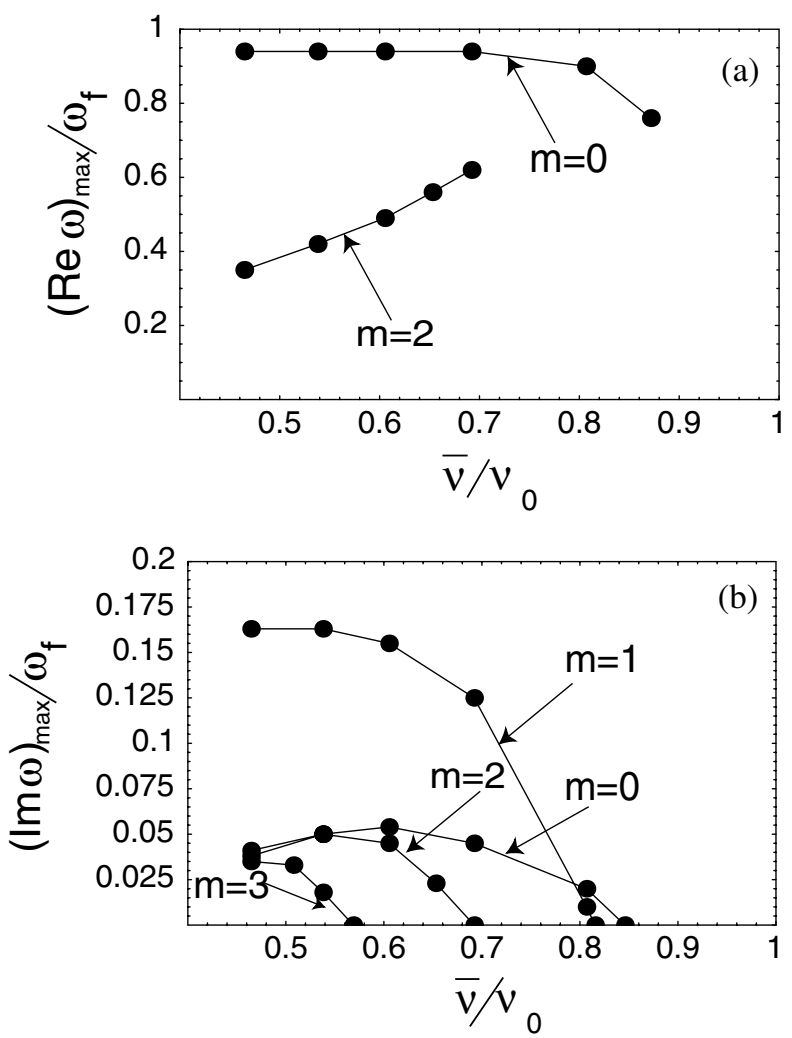

FIG. 8. Plots of normalized real frequency $(\operatorname{Re} \omega)_{\max } / \omega_{f}$ and growth rate $(\operatorname{Im} \omega)_{\max } / \omega_{f}$ at maximum growth versus normalized tune depression $\bar{\nu} / \nu_{0}$ for $T_{\| b} / T_{\perp b}=0.02$.

growth is illustrated in Fig. 9 for $s_{b}=0.95$. The simulation results presented in Figs. 6-9 are in good qualitative agreement with the theoretical model presented in Sec. II (see Figs. 1-4).

The longitudinal threshold temperature $T_{\| b}^{\text {th }}$ normalized to the transverse temperature $T_{\perp b}$ for the onset of instability for the arbitrary value of azimuthal mode number $m$ is plotted in Fig. 10 versus normalized beam

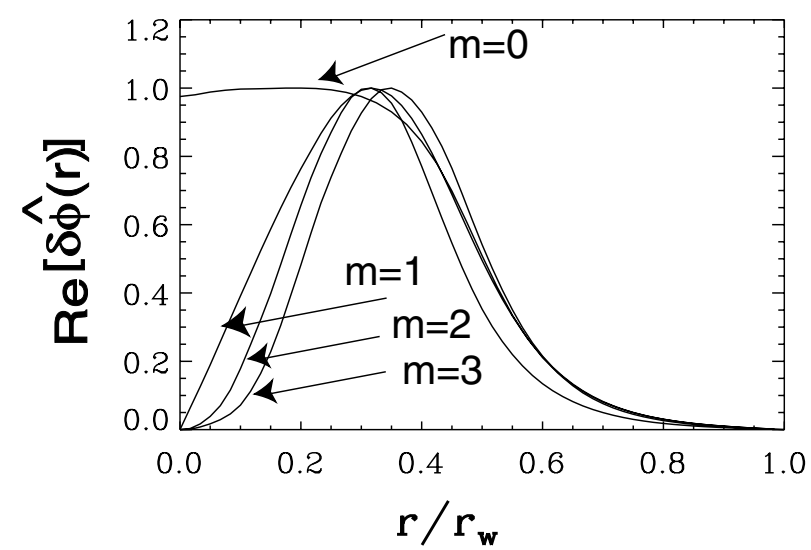

FIG. 9. Radial mode structure of the unstable $m=0,1,2,3$ eigenfunctions at maximum growth corresponding to $s_{b}=$ 0.95 and $T_{\| b} / T_{\perp b}=0.02$. intensity $s_{b}$ [Fig. 10(a)] and effective tune depression $\bar{\nu} / \nu_{0}$ [Fig. 10(b)]. The threshold temperature $T_{\| b}^{\text {th }}$ is a decreasing function of the normalized tune depression $\bar{\nu} / \nu_{0}$ and an increasing function of the normalized beam intensity $s_{b}$. Note from Fig. 10 that instability is absent for $T_{\| b} / T_{\perp b}>0.08$. The theoretical model in Sec. II predicts higher threshold temperatures than the simulation results in Fig. 10 and is less accurate in predicting the threshold temperature for the onset of instability.

Figures 11 and 12 show typical simulation results using the nonlinear version of the 3D BEST code for the case of normalized beam intensity $s_{b}=0.8$. In Fig. 11, the initial perturbation has a dominant initial excitation with $m=1$ and $k_{z} r_{w}=9$, and the time history of the perturbed density $\delta n_{b}=\int d^{3} p \delta f_{b}$ is plotted versus $\omega_{f} t$ at fixed axial position $z$ and radius $r=0.3 r_{b}$. After the initial linear growth phase, note from Fig. 11 that the instability saturates at a moderately large level with $\left|\delta n_{b}^{\max } / \hat{n}_{b}\right| \simeq 0.1$.

Shown in Fig. 12 is a plot of the average longitudinal momentum distribution $F_{b}\left(p_{z}, t\right)=\int d^{2} p_{\perp} d^{3} x f_{b}$ versus $p_{z}$ for a dominant initial excitation with $m=1$ and $k_{z} r_{w}=9$ (the case shown in Fig. 11). In Fig. 12, the
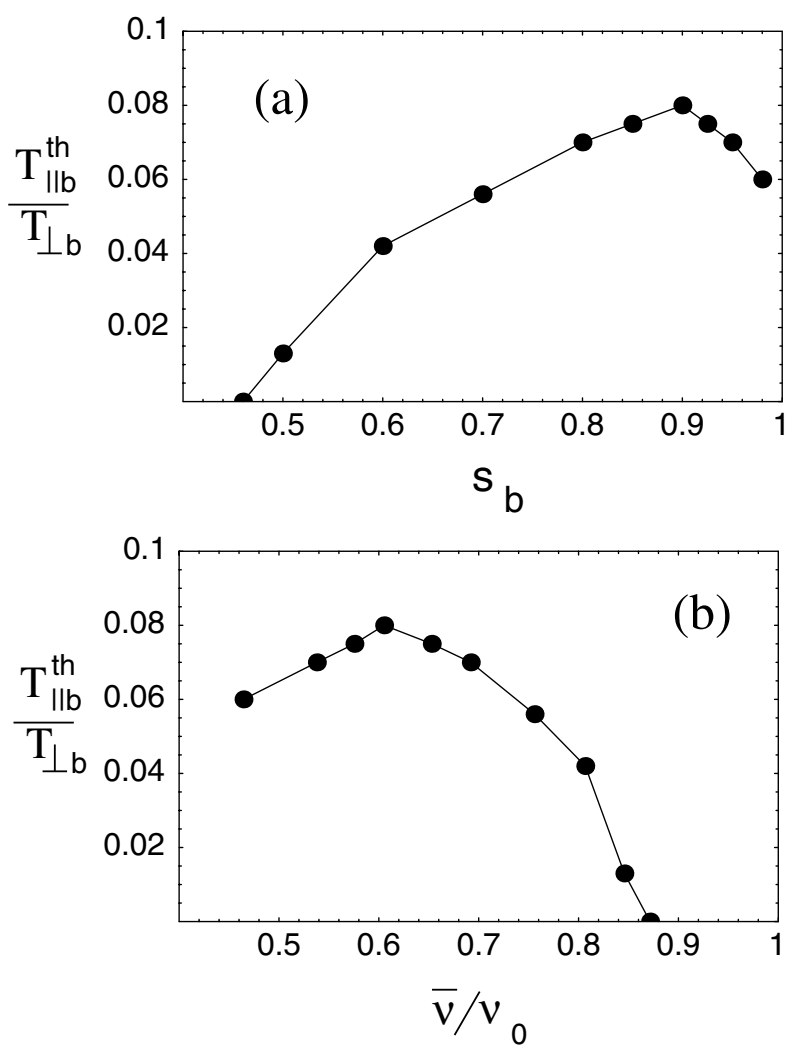

FIG. 10. Longitudinal threshold temperature $T_{\| b}^{\text {th }}$ normalized to the transverse temperature $T_{\perp b}$ for the onset of instability plotted versus (a) normalized beam intensity $s_{b}$ and (b) normalized tune depression $\bar{\nu} / \nu_{0}$. 


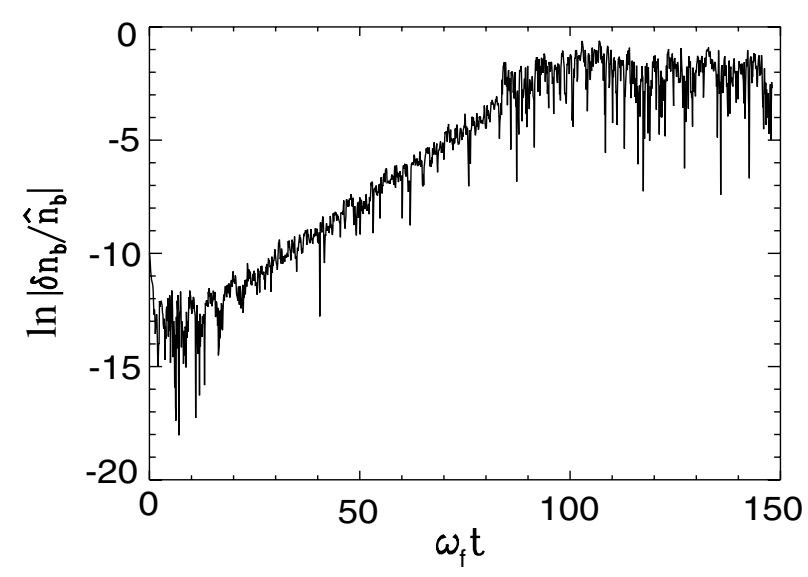

FIG. 11. Time history of the normalized density perturbation $\delta n_{\max } / \hat{n}_{b}$ for $s_{b}=0.8$ and $T_{\| b} / T_{\perp b}=0.02$ at fixed axial position $z$ and radius $r=0.3 r_{b}$.

average distribution $F_{b}\left(p_{z}, t\right)$ at time $t=150 \omega_{f}^{-1}$ (thick curve) is compared with the initial distribution (thin curve). The formation of tails in axial momentum space (Fig. 12) in the range of phase velocities of the unstable waves is a characteristic feature of resonant wave-particle interactions [1] which often leads to nonlinear saturation of collective instabilities. Therefore, the saturation of the instability is attributed to quasilinear stabilization due to resonant wave-particle interactions.

Moreover, the effective temperature ratio $T_{\| b} / T_{\perp b}$ for which the instability saturates can be estimated from the following arguments. As one can see from Fig. 12, the instability saturates due to the formation of tails in axial momentum space at resonant velocities $v_{\text {res }}=$ $\omega\left(k_{z}^{\max }\right) / k_{z}^{\max }$, where $\omega\left(k_{z}^{\max }\right)$ is the frequency of the most unstable mode with longitudinal wave number $k_{z}^{\max }$. Therefore, the width of the longitudinal velocity distribution after saturation is $T_{\| b} / T_{\perp b} \simeq v_{\mathrm{th} \|}^{2} / 2 r_{b}^{2} \omega_{\beta \perp}^{2}=$

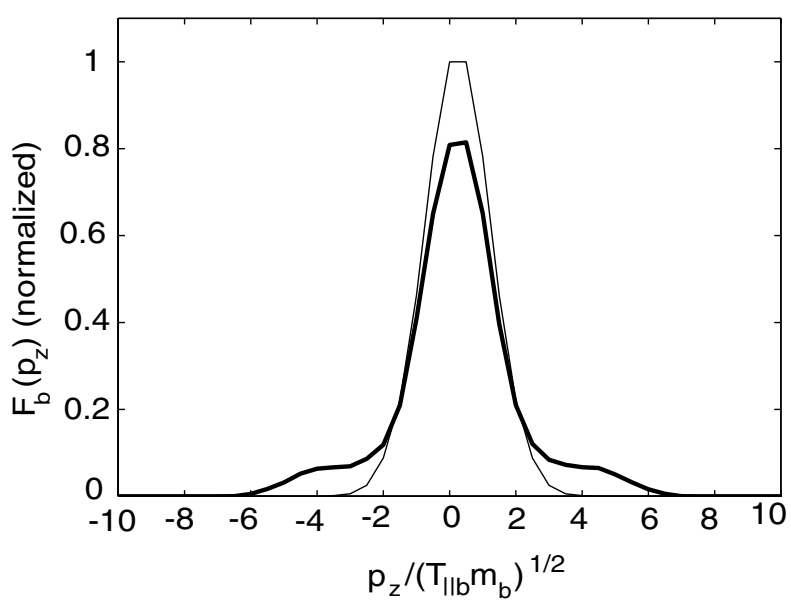

FIG. 12. Plot of average longitudinal momentum distribution $F_{b}\left(p_{z}, t\right)$ at time $t=0$ (thin line) and $t=150 \omega_{f}^{-1}$ (thick line), for normalized beam intensity $s_{b}=0.8$ and $T_{\| b} / T_{\perp b}=0.02$. $\left[\omega\left(k_{z}^{\max }\right) / \omega_{f}\right]^{2}\left(r_{w} / r_{b}\right)^{2}\left(\nu_{0} / \bar{\nu}\right) / 2\left(k_{z}^{\max } r_{w}\right)^{2}$, where use has been made of Eqs. (25) and (34). From Figs. 6 and 8, $k_{z}^{\max } r_{w} \approx 9, r_{w} / r_{b}=3, \omega\left(k_{z}^{\max }\right) / \omega_{f} \approx 0.9, \bar{\nu} / \nu_{0} \approx 0.5$, and the effective temperature ratio for which the instability saturates is $T_{\| b} / T_{\perp b} \approx 0.09$. This estimate is consistent with the threshold estimate in Fig. 10. In the simulations it is found that the $m=0$ mode saturates at the highest value of $T_{\| b} / T_{\perp b}$. Given the estimate above, since the $m=0$ mode has the highest phase velocity it may be the mode that determines the final velocity spread in the longitudinal direction. A more detailed investigation of the nonlinear saturation mechanism will be the subject of a future study.

Finally, the results for axisymmetric perturbations are similar to the results previously obtained by the authors [11]. For a discussion and comparison with previous work which studies the temperature anisotropy instability for axisymmetric perturbations about a KV distribution, the reader is referred to Ref. [11].

\section{CONCLUSIONS}

To summarize, in Sec. II we generalized the classical Harris-like instability to the case of an intense charged particle beam with anisotropic temperature $\left(T_{\| b} / T_{\perp b}<1\right)$ including the important effects of finite transverse geometry and beam space charge. Using the simplified assumption of negligible spread in depressed betatron frequency, we derived a simple dispersion equation for the lowest-order three-dimensional eigenmodes of the form $\phi_{n}(r) \exp \left(-i \omega t+i k_{z} z+i m \theta\right)$. For sufficiently large values of $k_{z}^{2} r_{b}^{2} \geq 1$, where $r_{b}$ is the rms beam radius, the analysis of the dispersion equation leads to a strong anisotropy-driven instability provided the normalized beam intensity $s_{b}=\hat{\omega}_{p b}^{2} / 2 \omega_{f}^{2}$ is sufficiently large. In Sec. IV, the BEST code [15], which implements the nonlinear $\delta f$ scheme described in Sec. III, was used to investigate the detailed stability properties of intense charged particle beams with large temperature anisotropy $\left(T_{\| b} / T_{\perp b} \ll 1\right)$ for three-dimensional perturbations with several values of azimuthal wave number $m=$ $0,1,2,3$. The simulation results clearly show that moderately intense beams with $s_{b} \gtrsim 0.5$ are linearly unstable to short-wavelength perturbations with $k_{z}^{2} r_{b}^{2} \gtrsim 1$, provided the ratio of longitudinal and transverse temperatures is smaller than some threshold value. The mode structure, growth rate, and conditions for the onset of the instability are qualitatively similar to what is predicted by the simple theoretical model presented in Sec. II. Both the simulations and the analytical theory predict that the dipole mode (azimuthal mode number $m=1$ ) is the most unstable mode. The main saturation mechanism for the instability is the resonant wave-particle interactions that occur during the formation of tails in the axial momentum distribution function. In the nonlinear saturation stage, the total distribution function is still far 
from equipartitioned, and free energy is still available to drive an instability of the hydrodynamic type [23,24].

\section{ACKNOWLEDGMENTS}

This research was supported by the U.S. Department of Energy. It is a pleasure to acknowledge the benefit of useful discussions with Igor Kaganovich, Irving Haber, Ingo Hofmann, and Steve Lund.

\section{APPENDIX A: EVALUATION OF ORBIT INTEGRAL}

In this Appendix, we evaluate the orbit integral [see Eq. (19)]

$$
\begin{gathered}
I_{n}^{p, m}=\int_{0}^{T_{r}} \frac{d \tau}{T_{r}} J_{m}[a \hat{r}(\tau)] \exp \left[i m \theta^{\prime}(\tau)-i m \omega_{\theta} \tau\right. \\
\left.-i p \omega_{r} \tau\right] .
\end{gathered}
$$

$P_{\theta}$ moving in the quadratic potential $\psi(r)=m_{b} \omega_{\beta \perp}^{2} r^{2} / 2$ with initial conditions $\hat{r}(\tau=0)=r_{\min }\left(H_{\perp}, P_{\theta}\right)$ and $\theta^{\prime}(\tau=0)=0$ [see Eq. (9)]. Here, $\omega_{r}\left(H_{\perp}, P_{\theta}\right)$ is the frequency of radial motion, and $\omega_{\theta}\left(H_{\perp}, P_{\theta}\right)$ is the angular frequency. In Cartesian coordinates, the particle trajectory $\{\hat{x}(\tau), \hat{y}(\tau)\}$ can be expressed as

$$
\begin{gathered}
\hat{x}(\tau)=\frac{\sqrt{H_{\perp} / m_{b}}}{\omega_{\beta \perp}} \cos \left(\omega_{\beta \perp} \tau\right)[\cos (\beta)-\sin (\beta)], \\
\hat{y}(\tau)=\frac{\sqrt{H_{\perp} / m_{b}}}{\omega_{\beta \perp}} \sin \left(\omega_{\beta \perp} \tau\right)[\sin (\beta)+\cos (\beta)],
\end{gathered}
$$

where $\cos (2 \beta)=P_{\theta} \omega_{\beta \perp} / H_{\perp}$. Therefore, we can express

$$
\begin{aligned}
\hat{r}^{2}(\tau) & =\hat{x}^{2}(\tau)+\hat{y}^{2}(\tau) \\
& =\frac{H_{\perp}}{m_{b} \omega_{\beta \perp}^{2}}\left[1-\sin (2 \beta) \cos \left(2 \omega_{\beta \perp} \tau\right)\right],
\end{aligned}
$$

and

$$
\hat{r}^{m}(\tau) \exp \left[i m \theta^{\prime}(\tau)\right]=[\hat{x}(\tau)+i \hat{y}(\tau)]^{m}=\left(\frac{H_{\perp}}{m_{b} \omega_{\beta \perp}^{2}}\right)^{m / 2}\left[\cos (\beta) \exp \left(i \omega_{\beta \perp} \tau\right)-\sin (\beta) \exp \left(-i \omega_{\beta \perp} \tau\right)\right]^{m} .
$$

From Eqs. (A2) and (A3), it follows that $\omega_{r}\left(H_{\perp}, P_{\theta}\right)=2 \omega_{\beta \perp}$ and $\omega_{\theta}\left(H_{\perp}, P_{\theta}\right)=\omega_{\beta \perp}$. Substituting Eqs. (A3) and (A4) into Eq. (A1) and changing the integration variable to $\alpha=2 \omega_{\beta \perp} \tau$, we obtain

$$
I_{n}^{p, m}=\int_{0}^{2 \pi} \frac{d \alpha}{2 \pi} J_{m}\left(a \frac{\sqrt{H_{\perp} / m_{b}}}{\omega_{\beta \perp}} \sqrt{1-\sin (2 \beta) \cos (\alpha)}\right) \exp [-i p \alpha-i(m / 2) \alpha] \frac{[\cos (\beta) \exp (i \alpha / 2)-\sin (\beta) \exp (-i \alpha / 2)]^{m}}{[1-\sin (2 \beta) \cos (\alpha)]^{m / 2}}
$$

Since the integral in Eq. (A5) is over one complete period of the integrand function $F(\alpha)$, it follows that

$$
\begin{aligned}
& I_{n}^{p, m}= \int_{0}^{2 \pi} d \alpha F(\alpha)=\int_{-\pi}^{\pi} d \alpha F(\alpha)=\int_{0}^{\pi} d \alpha\left[F(\alpha)+F^{*}(\alpha)\right] \\
&=\int_{0}^{\pi} \frac{d \alpha}{2 \pi} \frac{J_{m}\left[a \frac{\sqrt{H_{\perp} / m_{b}}}{\omega_{\beta \perp}} \sqrt{1-\sin (2 \beta) \cos (\alpha)}\right]}{[1-\sin (2 \beta) \cos (\alpha)]^{m / 2}}\{\exp (-i p \alpha-i(m / 2) \alpha)[\cos (\beta) \exp (i \alpha / 2) \\
&\left.-\sin (\beta) \exp (-i \alpha / 2)]^{m}+\text { c.c. }\right\} .
\end{aligned}
$$

To evaluate the integral in Eq. (A6), we use the summation theorem for Bessel functions [39], which gives

$$
J_{l}\left(w \sqrt{r^{2}+\rho^{2}-2 r \rho \cos \alpha}\right)=\exp (-i l \phi) \sum_{k=-\infty}^{+\infty} J_{k}(w \rho) J_{k+l}(w r) \exp (i k \alpha)=\exp (i l \phi) \sum_{k=-\infty}^{+\infty} J_{k}(w \rho) J_{k+l}(w r) \exp (-i k \alpha)
$$

where

$$
\exp (-i l \phi)=\left[\frac{r / \rho-\exp (i \alpha)}{r / \rho-\exp (-i \alpha)}\right]^{l / 2}
$$

In the present analysis, $w=a \sqrt{H_{\perp} / m_{b}} / \omega_{\perp \beta}, r=\sin \beta$, and $\rho=\cos \beta$, and therefore Eq. (A8) gives

$$
\exp (-i m \phi)=\exp (m \alpha / 2)\left[\frac{\cos \beta-\sin \beta \exp (i \alpha)}{\cos \beta-\sin \beta \exp (-i \alpha)}\right]^{m / 2} \text {. }
$$

Substituting Eqs. (A7)-(A9) into Eq. (A6) and rearranging terms, we obtain the expression 


$$
\begin{aligned}
I_{n}^{p, m} & =\sum_{k=-\infty}^{+\infty} J_{k}\left(a \frac{\sqrt{H_{\perp} / m_{b}}}{\omega_{\beta \perp}} \sin \beta\right) J_{k+m}\left(a \frac{\sqrt{H_{\perp} / m_{b}}}{\omega_{\beta \perp}} \cos \beta\right) \int_{0}^{\pi} \frac{d \alpha}{\pi} \cos [(k-p) \alpha] \\
& =J_{p}\left(a \frac{\sqrt{H_{\perp} / m_{b}}}{\omega_{\beta \perp}} \sin \beta\right) J_{p+m}\left(a \frac{\sqrt{H_{\perp} / m_{b}}}{\omega_{\beta \perp}} \cos \beta\right) \\
& =J_{p}\left(\frac{a}{\omega_{\beta \perp}} \sqrt{\frac{H_{\perp}+P_{\theta} \omega_{\beta \perp}}{2 m_{b}}}\right) J_{p+m}\left(\frac{a}{\omega_{\beta \perp}} \sqrt{\frac{H_{\perp}-P_{\theta} \omega_{\beta \perp}}{2 m_{b}}}\right) .
\end{aligned}
$$

\section{APPENDIX B: EVALUATION OF SUSCEPTIBILITY INTEGRAL}

To complete the evaluation of the susceptibility defined in Eq. (17), we determine the following integral:

$$
I=\frac{1}{m_{b} \lambda_{d}^{2}} \int_{0}^{\infty} \frac{d H_{\perp}}{T_{\perp b}} \int_{-H_{\perp} / \omega_{\beta \perp}}^{H_{\perp} / \omega_{\beta \perp}} \frac{d P_{\theta}}{2 \omega_{\beta \perp}} \exp \left[-\frac{H_{\perp}}{T_{\perp b}}\right]\left(I_{n}^{p, m}\right)^{*} I_{n^{\prime}}^{p, m}
$$

where the orbit integral $I_{n^{\prime}}^{p, m}$ is defined in Eq. (19) and was evaluated in Appendix A. Using Eqs. (19) and (A10), and introducing the change of integration variables,

$$
e_{+}=\left(H_{\perp}+P_{\theta} \omega_{\beta \perp}\right) / 2, \quad e_{-}=\left(H_{\perp}-P_{\theta} \omega_{\beta \perp}\right) / 2,
$$

Eq. (B1) can be rewritten as

$$
\begin{aligned}
I= & \frac{1}{m_{b} \lambda_{d}^{2} \omega_{\beta \perp}^{2} T_{\perp}} \int_{0}^{\infty} d e_{+} \int_{0}^{\infty} d e_{-} \exp \left[-\frac{e_{+}}{T_{\perp}}\right] \exp \left[-\frac{e_{-}}{T_{\perp}}\right] J_{p}\left(\frac{\lambda_{n^{\prime}} \sqrt{e_{-} / m_{b}}}{r_{w} \omega_{\beta \perp}}\right) J_{p+m}\left(\frac{\lambda_{n^{\prime}} \sqrt{e_{+} / m_{b}}}{r_{w} \omega_{\beta \perp}}\right) J_{p}\left(\frac{\lambda_{n} \sqrt{e_{-} / m_{b}}}{r_{w} \omega_{\beta \perp}}\right) \\
& \times J_{p+m}\left(\frac{\lambda_{n} \sqrt{e_{+} / m_{b}}}{r_{w} \omega_{\beta \perp}}\right) .
\end{aligned}
$$

The change of variables reduces the two-dimensional integral in Eq. (B1) to the product of two one-dimensional integrals. Using Eq. (25), the integral in Eq. (B3) can be evaluated as

$$
I=\frac{1}{2}\left(\frac{r_{b}}{\lambda_{d}}\right)^{2} I_{p}\left[\left(\frac{r_{b}}{r_{w}}\right)^{2} \frac{\lambda_{n} \lambda_{n^{\prime}}}{4}\right] I_{p+m}\left[\left(\frac{r_{b}}{r_{w}}\right)^{2} \frac{\lambda_{n} \lambda_{n^{\prime}}}{4}\right] \exp \left[-\left(\frac{r_{b}}{r_{w}}\right)^{2} \frac{\lambda_{n}^{2}+\lambda_{n^{\prime}}^{2}}{4}\right]
$$

where $r_{b}^{2}=2 T_{\perp b} / m_{b} \omega_{\beta \perp}^{2}$. The integral in Eq. (18) is

$$
q_{n, n^{\prime}}=\int_{0}^{1} d x x \exp \left[-\frac{r_{w}^{2} x^{2}}{r_{b}^{2}}\right] J_{m}\left(\lambda_{n} x\right) J_{m}\left(\lambda_{n^{\prime}} x\right)=\frac{1}{2}\left(\frac{r_{b}}{r_{w}}\right)^{2} I_{m}\left[\left(\frac{r_{b}}{r_{w}}\right)^{2} \frac{\lambda_{n} \lambda_{n^{\prime}}}{2}\right] \exp \left[-\left(\frac{r_{b}}{r_{w}}\right)^{2} \frac{\lambda_{n}^{2}+\lambda_{n^{\prime}}^{2}}{4}\right] .
$$

In Eqs. (B3) and (B5), we have extended the integration limits to infinity. The error in this approximation is proportional to $\exp \left[-\left(r_{w} / r_{b}\right)^{2}\right]$, which is small for $r_{w} / r_{b} \gtrsim 3$. Collecting the results from Eqs. (B4) and (B5), the expression for the matrix elements becomes

$$
\begin{aligned}
D_{n, n^{\prime}}^{m}=\frac{J_{m+1}^{2}\left(\lambda_{n}\right)}{2}\left(\lambda_{n}^{2}+k_{z}^{2} r_{w}^{2}\right) \delta_{n, n^{\prime}} & \\
+\frac{\hat{\omega}_{p}^{2}}{\omega_{\beta \perp}^{2}} \exp \left(-z_{n n}-z_{n^{\prime} n^{\prime}}\right)[ & I_{m}\left(2 z_{n n^{\prime}}\right)-\sum_{q=-\infty}^{+\infty} I_{q}\left(z_{n n^{\prime}}\right) I_{q+m}\left(z_{n n^{\prime}}\right) \int d v_{z} f_{0}\left(v_{z}\right)\left(\frac{\omega-k_{z} v_{z}}{\omega-(2 q+m) \omega_{\beta \perp}-k_{z} v_{z}}\right. \\
& \left.\left.+\frac{k_{z}^{2} v_{\mathrm{th} \perp}^{2}}{2\left[\omega-(2 q+m) \omega_{\beta \perp}-k_{z} v_{z}\right]^{2}}\right)\right],
\end{aligned}
$$

where $r_{b}^{2} / \lambda_{d}^{2}=2 \hat{\omega}_{p}^{2} / \omega_{\beta \perp}^{2}, v_{\mathrm{th} \perp}^{2}=2 T_{\perp b} / m_{b}$, and we have introduced $z_{n, n^{\prime}}=\left(r_{b} / r_{w}\right)^{2} \lambda_{n} \lambda_{n^{\prime}} / 4$. The terms in the summation in Eq. (B6) can be rearranged for even or odd values of azimuthal mode number $m$. We obtain 


$$
\begin{aligned}
& D_{n, n^{\prime}}^{m=2 l}= \frac{J_{m+1}^{2}\left(\lambda_{n}\right)}{2}\left(\lambda_{n}^{2}+k_{z}^{2} r_{w}^{2}\right) \delta_{n, n^{\prime}} \\
&+\frac{\hat{\omega}_{p}^{2}}{\omega_{\beta \perp}^{2}} \exp \left(-z_{n n}-z_{n^{\prime} n^{\prime}}\right)\left[I_{2 l}\left(2 z_{n n^{\prime}}\right)-I_{l}^{2}\left(z_{n n^{\prime}}\right) \int d v_{z} f_{0}\left(v_{z}\right)\left(1+\frac{k_{z}^{2} v_{\mathrm{th}}^{2}}{2\left(\omega-k_{z} v_{z}\right)^{2}}\right)\right. \\
&-\sum_{q>0} I_{q-l}\left(z_{n n^{\prime}}\right) I_{q+l}\left(z_{n n^{\prime}}\right) \int d v_{z} f_{0}\left(v_{z}\right)\left(\frac{2\left(\omega-k_{z} v_{z}\right)^{2}}{\left(\omega-k_{z} v_{z}\right)^{2}-4 q^{2} \omega_{\beta \perp}^{2}}\right. \\
&\left.\left.+k_{z}^{2} v_{\mathrm{th \perp}}^{2} \frac{\left(\omega-k_{z} v_{z}\right)^{2}+4 q^{2} \omega_{\beta \perp}^{2}}{\left[\left(\omega-k_{z} v_{z}\right)^{2}-4 q^{2} \omega_{\beta \perp}^{2}\right]^{2}}\right)\right]
\end{aligned}
$$

for even values of $m=2 l$, and

$$
\begin{aligned}
D_{n, n^{\prime}}^{m=2 l+1}=\frac{J_{m+1}^{2}\left(\lambda_{n}\right)}{2}\left(\lambda_{n}^{2}+k_{z}^{2} r_{w}^{2}\right) \delta_{n, n^{\prime}} & \\
+\frac{\hat{\omega}_{p}^{2}}{\omega_{\beta \perp}^{2}} \exp \left(-z_{n n}-z_{n^{\prime} n^{\prime}}\right)[ & I_{2 l+1}\left(2 z_{n n^{\prime}}\right) \\
& \quad-\sum_{q \geq 0} I_{q-l}\left(z_{n n^{\prime}}\right) I_{q+l+1}\left(z_{n n^{\prime}}\right) \int d v_{z} f_{0}\left(v_{z}\right)\left(\frac{2\left(\omega-k_{z} v_{z}\right)^{2}}{\left(\omega-k_{z} v_{z}\right)^{2}-(2 q+1)^{2} \omega_{\beta \perp}^{2}}\right. \\
& \left.\left.+k_{z}^{2} v_{\mathrm{th} \perp}^{2} \frac{\left(\omega-k_{z} v_{z}\right)^{2}+(2 q+1)^{2} \omega_{\beta \perp}^{2}}{\left[\left(\omega-k_{z} v_{z}\right)^{2}-(2 q+1)^{2} \omega_{\beta \perp}^{2}\right]^{2}}\right)\right],
\end{aligned}
$$

for odd values of $m=2 l+1$. If the distribution function $f_{0}\left(v_{z}\right)$ is Maxwellian with $f_{0}\left(v_{z}\right)=$ $\left(m_{b} / 2 \pi T_{\| b}\right)^{1 / 2} \exp \left(-m_{b} v_{z}^{2} / 2 T_{\| b}\right)$, then the matrix elements in Eqs. (B7) and (B8) take the form

$$
\begin{aligned}
D_{n, n^{\prime}}^{m=2 l}= & \frac{J_{m+1}^{2}\left(\lambda_{n}\right)}{2}\left(\lambda_{n}^{2}+k_{z}^{2} r_{w}^{2}\right) \delta_{n, n^{\prime}} \\
& +\frac{\hat{\omega}_{p}^{2}}{\omega_{\beta \perp}^{2}} \exp \left(-z_{n n}-z_{n^{\prime} n^{\prime}}\right)\left[I_{2 l}\left(2 z_{n n^{\prime}}\right)-I_{l}^{2}\left(z_{n n^{\prime}}\right) A_{0}(\omega)-\sum_{q>0} I_{q-l}\left(z_{n n^{\prime}}\right) I_{q+l}\left(z_{n n^{\prime}}\right)\left[A_{2 q}(\omega)+A_{-2 q}(\omega)\right]\right],
\end{aligned}
$$

for even $m=2 l$, and

$$
\begin{aligned}
D_{n, n^{\prime}}^{m=2 l+1}= & \frac{J_{m+1}^{2}\left(\lambda_{n}\right)}{2}\left(\lambda_{n}^{2}+k_{z}^{2} r_{w}^{2}\right) \delta_{n, n^{\prime}} \\
& +\frac{\hat{\omega}_{p}^{2}}{\omega_{\beta \perp}^{2}} \exp \left(-z_{n n}-z_{n^{\prime} n^{\prime}}\right)\left[I_{2 l+1}\left(2 z_{n n^{\prime}}\right)-\sum_{q \geq 0} I_{q-l}\left(z_{n n^{\prime}}\right) I_{q+l+1}\left(z_{n n^{\prime}}\right)\left[A_{2 q+1}(\omega)+A_{-2 q-1}(\omega)\right]\right],
\end{aligned}
$$

for odd $m=2 l+1$. Here

$$
A_{n}(\omega)=\left(1-\frac{v_{\mathrm{th} \perp}^{2}}{v_{\mathrm{th} \|}^{2}}\right)-Z\left(\frac{\omega-n \omega_{\beta \perp}}{k_{z} v_{\mathrm{th} \|}}\right)\left[\frac{\omega}{k_{z} v_{\mathrm{th} \|}}-\frac{\omega-n \omega_{\beta \perp}}{k_{z} v_{\mathrm{th} \|}}\left(1-\frac{v_{\mathrm{th} \perp}^{2}}{v_{\mathrm{th} \|}^{2}}\right)\right],
$$

where $Z(\Omega)$ is the plasma dispersion function $[1,40]$, and $v_{\mathrm{th} \|}=\left(2 T_{\| b} / m_{b}\right)^{1 / 2}$.

[1] R. C. Davidson and H. Qin, Physics of Intense Charged Particle Beams in High Energy Accelerators (World Scientific, Singapore, 2001), and references therein.

[2] M. Reiser, Theory and Design of Charged Particle Beams (John Wiley \& Sons, Inc., New York, 1994).

[3] A.W. Chao, Physics of Collective Beam Instabilities in High Energy Accelerators (John Wiley \& Sons, Inc., New York, 1993).
[4] See, for example, Proceedings of the 1999 International Heavy Ion Fusion Symposium [Nucl. Instrum. Methods Phys. Res., Sect. A 464, 1-674 (2001).

[5] See, for example, Proceedings of the 1999 Particle Accelerator Conference, New York (IEEE, Piscataway, NJ, 1999), pp. 1-3778.

[6] I. M. Kapchinskij and V.V. Vladimirskij, in Proceedings of the International Conference on High Energy Accelerators and Instrumentation, Geneva, 1959 (CERN Scientific Information Service, Geneva, 1959), p. 274.

[7] R. L. Gluckstern, in Proceedings of the 1970 Proton Linear Accelerator Conference, Batavia, IL, edited by M. R. Tracy (National Accelerator Laboratory, Batavia, IL, 1971), p. 811. 
[8] T.-S. Wang and L. Smith, Part. Accel. 12, 247 (1982).

[9] R. C. Davidson, Phys. Rev. Lett. 81, 991 (1998).

[10] R. C. Davidson and H. Qin, Phys. Rev. ST Accel. Beams 2, 114401 (1999).

[11] E. A. Startsev, R. C. Davidson, and H. Qin, Phys. Plasmas 9, 3138 (2002).

[12] E. A. Startsev, R. C. Davidson, and H. Qin, Laser Part. Beams 20, 585 (2002).

[13] Q. Qian, W.W. Lee, and R. C. Davidson, Phys. Plasmas 4, 1915 (1997).

[14] W.W. Lee, Q. Qian, and R. C. Davidson, Phys. Lett. A 230, 347 (1997).

[15] H. Qin, R. C. Davidson, and W.W. Lee, Phys. Rev. ST Accel. Beams 3, 084401 (2000); 3, 109901 (2000).

[16] P. H. Stoltz, R. C. Davidson, and W. W. Lee, Phys. Plasmas 6, 298 (1999).

[17] H. Qin, R. C. Davidson, W.W. Lee, and E. A. Startsev, in Proceedings of the 2001 Particle Accelerator Conference, Chicago, IL (IEEE, Piscataway, NJ, 2001), p. 693.

[18] I. Hofmann, L. J. Laslett, L. Smith, and I. Haber, Part. Accel. 13, 145 (1983)

[19] E. A. Startsev, R. C. Davidson, W.W. Lee, and H. Qin, in Proceedings of the 2001 Particle Accelerator Conference, Chicago, IL (Ref. [17]), p. 3081.

[20] S. M. Lund, J. J. Barnard, G. D. Craig, A. Friedman, D. P. Grote, T. S. Sangster, W. M. Sharp, S. Eylon, T. T. Fessenden, E. Henestroza, S. Yu, and I. Haber, Nucl. Instrum. Methods Phys. Res., Sect. A 415, 345 (1998).

[21] A. Friedman, J. J. Barnard, D. P. Grote, and I. Haber, Nucl. Instrum. Methods Phys. Res., Sect. A 415, 455 (1998).

[22] S. M. Lund and R. C. Davidson, Phys. Plasmas 5, 3028 (1998).

[23] S. Strasburg and R. C. Davidson, Phys. Lett. A 269, 40 (2000).

[24] I. Hofmann, Phys. Rev. E 57, 4713 (1998).
[25] R. C. Davidson and S. Strasburg, Phys. Plasmas 7, 2657 (2000).

[26] E. G. Harris, Phys. Rev. Lett. 2, 34 (1959).

[27] A. B. Mikhailovskii, Theory of Plasma Instabilities (Consultants Bureau, New York, 1974), Vol. 1, pp. 183197.

[28] I. Haber (private communication).

[29] A. Friedman (private communication).

[30] A. Friedman, D. A. Callahan, D. P. Grote, and A. B. Langdon, Bull. Am. Phys. Soc. 35, 2121 (1990).

[31] A. Friedman, R. O. Bangerter, D. A. Callahan, D. P. Grote, and A. B. Langdon, in Proceedings of the 2nd European Particle Accelerator Conference, Nice, France, 1990, edited by P. A. Martin (Editions Frontíers, Gif-sur-Yvette, France, 1990), Vol. 2, p. 1699.

[32] A. Friedman, D. P. Grote, and I. Haber, Phys. Fluids B 4, 2203 (1992).

[33] S. M. Lund, D. A. Callahan, A. Friedman, D. P. Grote, I. Haber, and T.F. Wang, in Proceedings of the XIX International Linear Accelerator Conference, Chicago, 1998, edited by C. E. Eyberger, R. C. Pardo, and M. M. White (Argonne National Laboratory, Argonne, IL, 1998), p. 372.

[34] I. Haber, A. Friedman, D. P. Grote, S. M. Lund, and R. A. Kishek, Phys. Plasmas 6, 2254 (1999).

[35] I. Hofmann, J. Qiang, and R. Ryne, Phys. Rev. Lett. 86, 2313 (2001).

[36] I. Hofmann, G. Franchetti, O. Boine-Frankenheim, J. Qiang, and R. D. Ryne, Phys. Rev. ST Accel. Beams 6, 024202 (2003).

[37] S. E. Parker and W.W. Lee, Phys. Fluids B 5, 77 (1993).

[38] R. C. Davidson, W.W. Lee, H. Qin, and E. A. Startsev, Phys. Plasmas 9, 340 (2002).

[39] I. S. Gradshteyn and I. M. Ryzhik, Tables of Integrals, Series, and Products (Academic Press, New York, 1965).

[40] NRL Plasma Formulary (Naval Research Laboratory, Washington, DC, 1998). 